\title{
Associations between the von Willebrand Factor -ADAMTS13 Axis, Complement Activation, and COVID-19 Severity and Mortality
}

\author{
György Sinkovits ${ }^{1}$ Marienn Réti ${ }^{2}$ Veronika Müller ${ }^{3}$ Zsolt Iványi ${ }^{4}$ János Gál ${ }^{4}$ László Gopcsa ${ }^{2}$ \\ Péter Reményi ${ }^{2}$ Beáta Szathmáry ${ }^{5}$ Botond Lakatos ${ }^{5}$ János Szlávik ${ }^{5}$ Ilona Bobek ${ }^{6}$ \\ Zita Z. Prohászka ${ }^{1}$ Zsolt Förhécz ${ }^{1}$ Blanka Mező ${ }^{1,7}$ Dorottya Csuka ${ }^{1}$ (i) Lisa Hurler $^{1}$ (1) Erika Kajdácsi $^{1}$ \\ László Cervenak $^{1}$ Petra Kiszel ${ }^{7}$ Tamás Masszi ${ }^{1} \quad$ István Vályi-Nagy ${ }^{2, *}$ Zoltán Prohászka ${ }^{1,7, *}$
}

\footnotetext{
${ }^{1}$ Department of Internal Medicine and Haematology, Semmelweis University, Budapest, Hungary

${ }^{2}$ Department of Haematology and Stem Cell Transplantation, Central Hospital of Southern Pest, Institute of Haematology and Infectious Diseases, Budapest, Hungary

${ }^{3}$ Department of Pulmonology, Semmelweis University, Budapest, Hungary

${ }^{4}$ Department of Anaesthesiology and Intensive Therapy, Semmelweis University, Budapest, Hungary

${ }^{5}$ Department of Infectology, Central Hospital of Southern Pest, Institute of Haematology and Infectious Diseases, Budapest, Hungary
}

Address for correspondence György Sinkovits, MD, PhD, Department of Internal Medicine and Haematology, Semmelweis University, H-1088 Budapest, Szentkirályi St. 46, Hungary (e-mail: sinkovits.gyorgy@med.semmelweis-univ.hu).

\footnotetext{
${ }^{6}$ Department of Anaesthesiology and Intensive Therapy, Central Hospital of Southern Pest, Institute of Haematology and Infectious Diseases, Budapest, Hungary

${ }^{7}$ Research Group for Immunology and Haematology, Semmelweis University - Eötvös Loránd Research Network (Office for Supported Research Groups), Budapest, Hungary
}

\author{
Abstract \\ Keywords \\ - ADAMTS13 protein \\ - complement \\ activation \\ - COVID-19 \\ - survival analysis \\ - von Willebrand factor
}

Background Endothelial and complement activation were both associated with immunothrombosis, a key determinant of COVID-19 severity, but their interrelation has not yet been investigated.

Objectives We aimed to determine von Willebrand factor (VWF) antigen (VWF:Ag) concentration, VWF collagen binding activity (VWF:CBA), a disintegrin and metalloproteinase with a thrombospondin type 1 motif, member 13 (ADAMTS13) activity (ADAMTS13:Ac), and their ratios in hospitalized COVID-19 patients, and to investigate how these parameters and their constellation with complement activation relate to disease severity and in-hospital mortality in COVID-19.

Methods Samples of 102 hospitalized patients with polymerase chain reactionconfirmed severe acute respiratory syndrome coronavirus 2 positivity were included in our observational cohort study. Patients were stratified according to the peak severity of COVID-19 disease in agreement with the World Health Organization ordinal scale. Twenty-six convalescent plasma donors with previous COVID-19 disease formed

\footnotetext{
Shared authorship.
}

received

June 30, 2021

accepted after revision

October 10, 2021
DOI https://doi.org/

$10.1055 / \mathrm{s}-0041-1740182$. ISSN 0340-6245.

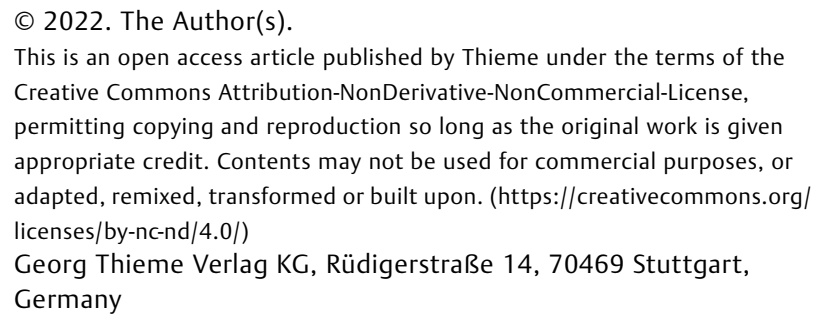




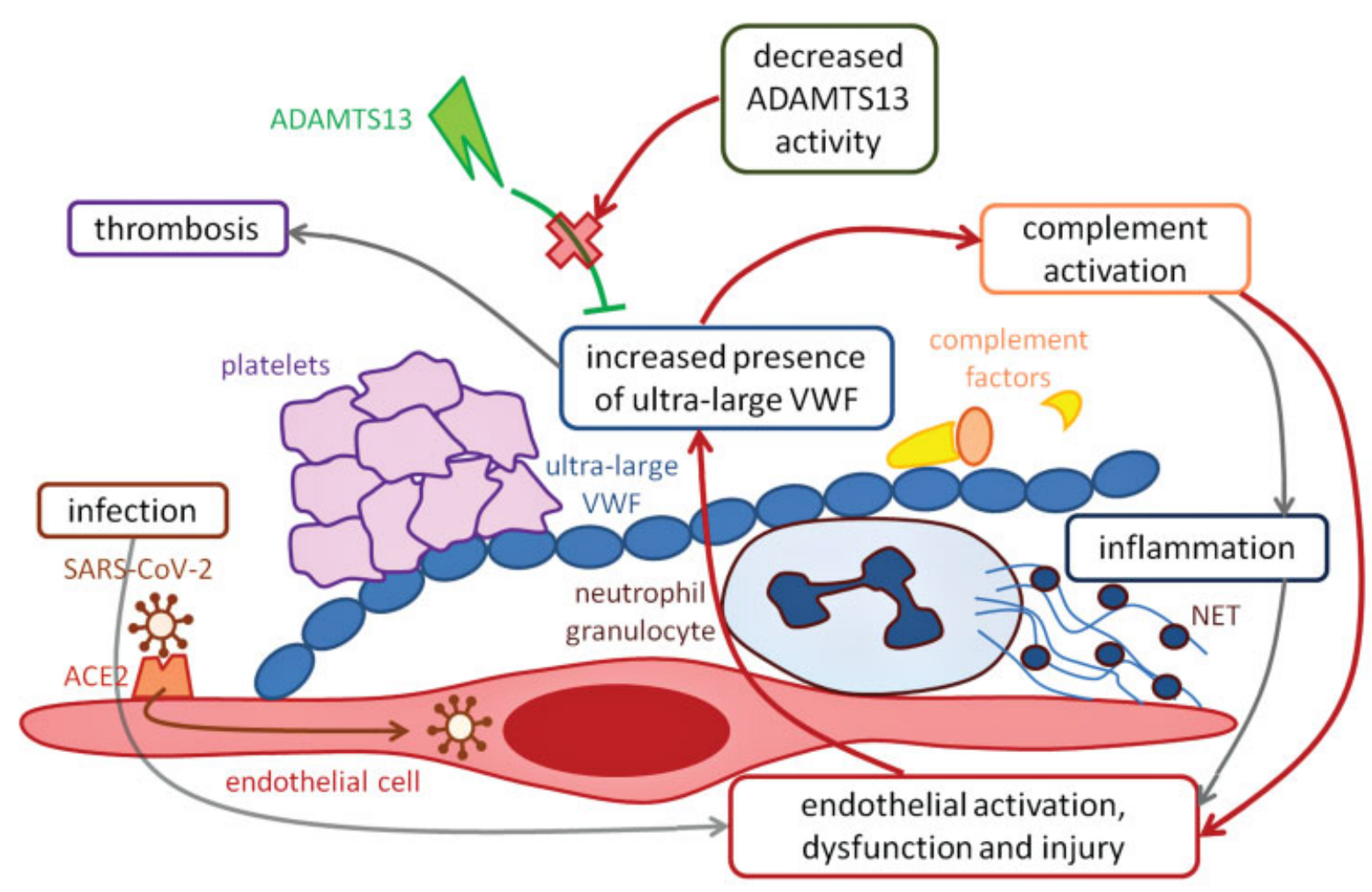

Visual summary. Scheme of potential interactions between the von Willebrand factor-a disintegrin and metalloproteinase with a thrombospondin type 1 motif, member 13 (VWF-ADAMTS13) axis and complement activation in COVID-19.

the control group. VWF:Ag concentration and VWF:CBA were determined by enzymelinked immunosorbent assay (ELISA); ADAMTS13:Ac was determined by fluorescence resonance energy transfer. Complement $\mathrm{C} 3$ and $\mathrm{C} 3$ a were measured by turbidimetry and ELISA, respectively. Clinical covariates and markers of inflammation were extracted from hospital records.

Results VWF:Ag and VWF:CBA were elevated in all groups of hospitalized COVID-19 patients and increased in parallel with disease severity. ADAMTS13:Ac was decreased in patients with severe COVID-19, with the lowest values in nonsurvivors. High (>300\%) VWF:Ag concentrations or decreased $(<67 \%)$ ADAMTS13:Ac were associated with higher risk of severe COVID-19 disease or in-hospital mortality. The concomitant presence of decreased ADAMTS13:Ac and increased C3a/C3 ratio-indicating complement overactivation and consumption-was a strong independent predictor of inhospital mortality.

Conclusion Our results suggest that an interaction between the VWF-ADAMTS13 axis and complement overactivation and consumption plays an important role in the pathogenesis of COVID-19.

\section{Introduction}

The coronavirus disease 2019 (COVID-19), caused by the severe acute respiratory syndrome coronavirus 2 (SARSCoV-2), presents in highly variable clinical forms ranging from a mild upper respiratory tract infection to severe respiratory failure necessitating mechanical ventilation. ${ }^{1,2}$ The disease primarily affects the respiratory system, however, especially in severe cases, multiple organ systems may be involved. ${ }^{1,2}$ In severe COVID-19, pathological overproduction of proinflammatory cytokines (termed cytokine storm) has been described; the consequent systemic 
hyperinflammation is responsible for most detrimental effects of the disease. ${ }^{3,4}$ In parallel with the proinflammatory changes, a prothrombotic state is also present, indicated by an increased risk of venous, arterial, and microvascular thrombotic events and by characteristic changes in laboratory parameters, such as elevated fibrinogen and D-dimer levels. ${ }^{1,2,5,6}$ The concentration of von Willebrand factor (VWF) is increased, ${ }^{7-10}$ whereas the activity of a disintegrin and metalloproteinase with a thrombospondin type 1 motif, member 13 (ADAMTS13) metalloprotease-responsible for cleaving ultra-large VWF multimers-is decreased, ${ }^{11-14}$ resulting in an imbalance of the VWF-ADAMTS13 axis, ${ }^{12,15-17}$ which was associated with a higher severity and mortality of COVID-19. ${ }^{11,14,18-21}$

Besides that of VWF, concentrations of further endothelial markers are also increased in COVID-19, 8,18,22,23 indicating a role of altered endothelial cell function in the pathogenesis of severe COVID-19 disease. Endothelial cells can directly be infected by the SARS-CoV-2 virus via their angiotensin-converting enzyme 2 receptors ${ }^{24}$; moreover, they are important target cells of inflammatory mediators, which are abundant in severe COVID-19. ${ }^{3,4}$ The consequential endothelial activation and dysfunction may result in hemostatic abnormalities, ${ }^{25}$ and in the dysregulation and overactivation of multiple plasma enzyme systems, including the complement system. ${ }^{26}$ The complement system was indeed found to be activated in COVID-19; the extent of complement activation was associated with the severity and outcome of the COVID-19 disease. ${ }^{27-29}$ Furthermore, a strong correlation was described between markers of endothelial and complement activation in COVID-19, ${ }^{22}$ which may reflect the fact that the two processes are linked on multiple levels: endothelial dysfunction facilitates complement activation, whereas complement anaphylatoxins and other activation products may in turn perturb endothelial function. ${ }^{30}$

Based on the above, we hypothesized that the pathological activation of endothelial cells and the complement system contribute jointly to the pathogenesis of the COVID-19 disease.

Accordingly, or aim was to determine the VWF antigen (VWF:Ag) concentration, VWF collagen binding activity (VWF:CBA), ADAMTS13 activity (ADAMTS13:Ac), and their ratios in hospitalized COVID-19 patients, and to investigate how these parameters and their constellations with markers of complement activation relate to disease severity and inhospital mortality in COVID-19.

\section{Methods}

\section{Patient Selection, Outcomes, and Definitions}

To enroll a cohort of adult (above 18 years of age) hospitalized COVID-19 patients, we screened and sampled 110 adult patients who were treated for suspected COVID-19 disease in two tertiary referral hospitals in Budapest between April 20 and July 2, 2020. One hundred and two of the above patients with confirmed COVID-19 infection-positive reverse transcription polymerase chain reaction (RT-PCR) test result for SARS-CoV-2 in at least one nasopharyngeal swab sample-were included in our study.

The enrolled hospitalized patients were categorized according to the maximal (peak) severity of the COVID-19 disease- and also according to the severity at sampling-in agreement with the World Health Organization (WHO) Ordinal Scale for Clinical Improvement (https://www.who.int/blueprint/ priority-diseases/key-action/COVID-19_Treatment_Trial_Design_ Master_Protocol_synopsis_Final_18022020.pdf). Patients who did not need oxygen therapy formed the HOSP (WHO-3: hospitalized, no oxygen therapy) subgroup. Those patients who received oxygen support, but did not require intubation and mechanical ventilation or admission to intensive care unit (ICU) formed the HOSP + O2 (WHO-4: oxygen by mask or nasal prongs) subgroup. The severity of the above cases was considered moderate, while fatal cases and cases requiring ICU admission were considered severe. Surviving severe patients constituted the ICU (WHO-6/7: intubation and mechanical ventilation \pm additional organ support) subgroup, whereas the deceased patients comprised the FATAL (WHO-8: death) severity subgroup.

Twenty-six volunteers, who were registered to donate convalescent plasma in a clinical trial and had evidence of a previous COVID-19 disease (positive SARS-CoV-2 RT-PCR at the time of the disease) not requiring hospitalization, were sampled and included in the convalescent phase as a patient control group. The scheme of patient and control subject enrolment is represented in -Supplementary Fig. $\mathbf{S 1}$ (available in the online version).

Digital hospital records were available for all enrolled patients; these were used for the collection of the necessary clinical, radiological, and basic laboratory data.

The study was conducted in accordance with the Declaration of Helsinki and its subsequent revisions, and was approved by the Hungarian Scientific and Research Ethics Committee (ETT-TUKEB; No. IV/4403-2/2020/EKU). Written informed consent was obtained from the patients and control subjects, or from the closest relative available, if the patient was unable to give informed consent.

\section{Samples}

Blood samples were drawn from the antecubital vein or from a central venous catheter, and were immediately transferred to the processing laboratory, where the cells and the supernatant-serum, citrate-, and ethylenediaminetetraacetic acid-anticoagulated plasma-were separated by centrifugation. Serum and plasma aliquots were immediately frozen and stored at $-70^{\circ} \mathrm{C}$ until measurements.

Only one sample per patient was included into the study, if more samples were available, the one taken at the most severe clinical stage was included. The median time from hospital admission until sample collection was 3 days (interquartile range: $1-7$ days).

\section{Laboratory Determinations}

ADAMTS13:Ac was determined by a fluorescence resonance energy transfer assay using the FRETS-VWF73 substrate, as described earlier. ${ }^{31}$

VWF:Ag concentration and VWF:CBA were measured by in-house sandwich enzyme-linked immunosorbent assay methods described earlier. ${ }^{32}$ 
Both parameters were expressed as percentages, where the ADAMTS13:Ac, VWF:Ag, and VWF:CBA value of a citrated plasma pool of healthy human individuals was regarded as $100 \%$. The VWF:Ag level of our citrated plasma pool was essentially similar $(1.033 \mathrm{IU} / \mathrm{mL})$ to that of a commercially available calibrator (TECHNOZYM vWF:Ag Calibrator Set, Technoclone GMBH, Vienna, Austria).

Determination of complement parameters was described earlier. ${ }^{29}$

Further laboratory data were extracted from hospital records.

\section{Statistical Analyses}

Categorical data are reported as frequencies (\%); chi-square and Fisher's exact tests were used to compare categorical data between groups. Most continuous variables showed skewed distributions, so these data were presented as median and interquartile range, and nonparametric tests were used: Mann-Whitney test for the comparison of two independent groups, Kruskal-Wallis test with Dunn's post-test for the comparison of more than two independent groups, and Spearman's rank correlation test for analyzing the correlations between continuous variables. Cases with missing data were excluded pairwise. Receiver operating characteristic (ROC) curves were generated and analyzed to determine optimal cutoff points for transforming continuous variables into binary categorical variables. Uni- and multivariable logistic regression models were built to assess the effects of predictor variables on disease severity, and uni- and multivariable Cox proportional hazard models were used to assess the effects of various clinical and laboratory parameters on in-hospital mortality. Survival was defined as time from hospitalization until the last follow-up visit before September 5, 2020, or until death (all-cause, in-hospital mortality). Kaplan-Meier curves were generated to show the occurrence of primary events plotted against time. Regression models were adjusted for a baseline model consisting of age, the number of comorbidities, and C-reactive protein (CRP) concentrations. The baseline model was the final, best-fitting model built in a conditional forward stepwise manner based on age, the number of comorbidities, and the following laboratory parameters associated with disease severity: lymphocyte count, CRP, D-dimer, and interleukin-6 (IL-6) levels. Statistical interaction, analyzed in Cox proportion hazard models, means that the association of a variable with another is dependent on a third variable. Statistical calculations were performed by GraphPad Prism 9 (GraphPad Softwares Inc., La Jolla, California, United States), Statistica (version 13.5.0.17, TIBCO Software Inc., Palo Alto, California, United States), and IBM SPSS Statistics 27 (IBM Corporation, Armonk, New York, United States) software.

\section{Results}

\section{Description of the Patient Cohort and Severity Subgroups}

A total of 102 hospitalized COVID-19 patients were enrolled in our study cohort ( - Supplementary Fig. S1, available in the online version). In addition, 26 plasma donors in the convalescent phase who were outpatients at the time of a previous SARS-CoV-2 infection (symptom onset median 54 [range: 26-74] days before sampling) were included as a patient control group (CONTR).

Hospitalized patients $(n=102)$ were divided into subgroups based on the peak disease severity (- Supplementary Fig. S1, available in the online version).

Twenty-seven patients did not need oxygen therapy during their hospital stay; these patients formed the HOSP subgroup. Thirty-three patients who received oxygen support, but did not require intubation and mechanical ventilation or admission to ICU, formed the HOSP $+\mathrm{O} 2$ subgroup. Thirty patients required intubation and mechanical ventilation, these and further eight patients were admitted to the ICU. Seventeen of the above patients survived, they composed the ICU subgroup. Twenty-five patients died during their hospital stay, the deceased patients comprised the FATAL subgroup. None of the patients in our cohort were treated by noninvasive ventilation or high-flow oxygen therapy (WHO-5).

Demographic, anamnestic, clinical, and laboratory parameters in the above outlined peak severity subgroups are summarized in -Table 1. (An alternative classification based on the disease severity at the time of sampling was also performed; the description and basic laboratory parameters of these groups are shown in -Supplementary Table 51, available in the online version.)

The patients' age and the number of comorbidities were higher in patients who later died, and several complications (respiratory failure, macrothromboembolic complications and acute kidney injury) were more frequent in severe cases (i.e., in patients who were treated in the ICU and/or died) compared with other patients.

Neutrophil granulocyte counts were higher, whereas lymphocyte counts were lower in severe cases. Markers and mediators of inflammation (CRP and IL-6) gradually increased in parallel with increasing severity of COVID-19.

Platelet counts were in the normal range or slightly decreased and did not differ significantly across severity subgroups or from patient controls. Prothrombin time showed a gradual increase in parallel with increasing disease severity. Thrombin time was prolonged in fatal cases. D-dimer levels were significantly elevated in all groups of hospitalized COVID19 patients in comparison to patient controls, with $90.2 \%$ of hospitalized COVID-19 patients' values above the upper limit of normal range. Fibrinogen levels showed a gradual increase across the HOSP, HOSP + O2, and ICU groups, with $81.2 \%$ of patients in the ICU group having elevated fibrinogen levels. However, there was a drop in fibrinogen levels in multiple fatal cases: $40.0 \%$ of patients in the FATAL group had normal or slightly decreased fibrinogen levels.

\section{von Willebrand Factor Antigen, Collagen Binding Activity, ADAMTS13 Activity, and Their Ratio in COVID-19 Disease}

-Fig. 1 shows VWF:Ag concentration, VWF:CBA, ADAMTS13: Ac, and their ratios in patients classified according to the peak severity of COVID-19 disease. 


\begin{tabular}{|c|c|c|c|c|c|c|c|c|c|c|c|c|c|c|c|c|c|c|c|c|c|}
\hline$\frac{\overbrace{0}^{0}}{\frac{0}{n}}$ & 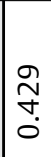 & $\begin{array}{l}\bar{\delta} \\
\dot{0} \\
\dot{0} \\
\vee\end{array}$ & & $\begin{array}{l}0 \\
0 \\
0 \\
0\end{array}$ & $\begin{array}{l}\infty \\
\bar{\sigma} \\
\end{array}$ & $\begin{array}{l}n \\
0 \\
0 \\
0\end{array}$ & $\begin{array}{l}0 \\
0 \\
0 \\
0\end{array}$ & $\underset{\Sigma}{\Sigma}$ & $\mid \begin{array}{l}m \\
0 \\
\dot{0}\end{array}$ & $\mid \begin{array}{l}n \\
\infty \\
\infty \\
0 \\
0\end{array}$ & & $\begin{array}{l}\stackrel{0}{m} \\
\stackrel{0}{0}\end{array}$ & & $\begin{array}{l}\bar{\delta} \\
\dot{0} \\
0 \\
v\end{array}$ & $\begin{array}{l}\overline{8} \\
0 \\
0 \\
\dot{0} \\
v\end{array}$ & Oे & & $\begin{array}{l}0 \\
0 \\
0 \\
0\end{array}$ & \begin{tabular}{|l}
$\bar{\delta}$ \\
$\overline{0}$ \\
0 \\
0 \\
$v$
\end{tabular} & $\begin{array}{l}\bar{\delta} \\
\bar{\delta} \\
0 \\
0 \\
v\end{array}$ & $\begin{array}{l}\bar{\delta} \\
\vdots \\
\dot{0} \\
v\end{array}$ \\
\hline 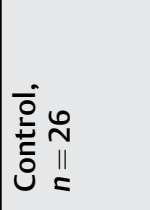 & 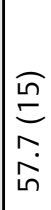 & 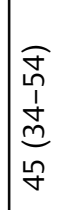 & & $\begin{array}{l}\widehat{c} \\
\bar{c} \\
0 \\
0\end{array}$ & 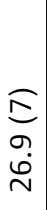 & $\begin{array}{l}0 \\
0 \\
0 \\
0\end{array}$ & $\begin{array}{l}\Xi \\
\infty \\
m \\
m\end{array}$ & $\begin{array}{l}0 \\
0 \\
0 \\
0\end{array}$ & $\begin{array}{l}0 \\
0 \\
0 \\
0\end{array}$ & $\begin{array}{l}\Xi \\
\infty \\
\dot{m} \\
\dot{m}\end{array}$ & & 1 & & $\begin{array}{l}0 \\
0 \\
0 \\
0\end{array}$ & $\begin{array}{l}0 \\
0 \\
0 \\
0\end{array}$ & $\begin{array}{l}\widehat{0} \\
0 \\
0 \\
0\end{array}$ & & $\begin{array}{l}\widehat{\sigma} \\
\dot{1} \\
\vdots \\
0 \\
\dot{m} \\
o \\
\tilde{m} \\
\dot{m}\end{array}$ & 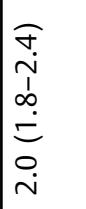 & 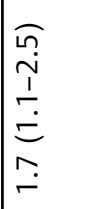 & 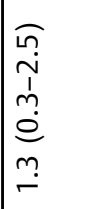 \\
\hline 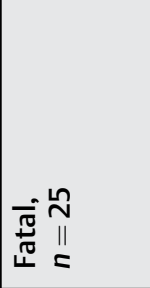 & 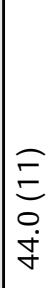 & $\begin{array}{l}0 \\
0 \\
1 \\
1 \\
\\
0 \\
0\end{array}$ & & $\begin{array}{l}\vec{f} \\
\stackrel{J}{d} \\
\stackrel{\sigma}{d}\end{array}$ & $\begin{array}{l}\widehat{O} \\
\stackrel{\Xi}{ } \\
\check{o} \\
\infty\end{array}$ & $\begin{array}{l}\hat{a} \\
0 \\
\dot{b} \\
m\end{array}$ & $\begin{array}{l}\tilde{\Xi} \\
\Xi \\
\check{f}\end{array}$ & $\begin{array}{l}\widehat{E} \\
\text { E } \\
\dot{\forall} \\
\dot{f}\end{array}$ & $\begin{array}{l}\widehat{a} \\
\overline{\dot{b}} \\
\dot{m}\end{array}$ & 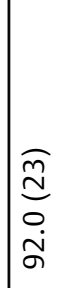 & & 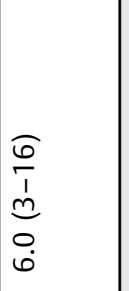 & & 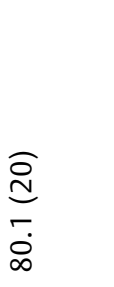 & $\begin{array}{l}\widehat{D} \\
0 \\
\infty \\
\infty\end{array}$ & $\begin{array}{l}\hat{a} \\
0 \\
\dot{0} \\
m\end{array}$ & & 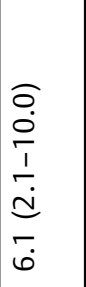 & 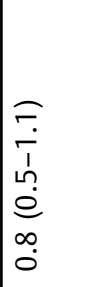 & 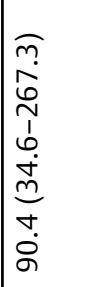 & 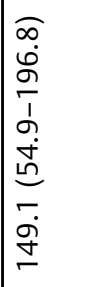 \\
\hline$\underline{\underline{\partial}}=$ & $\begin{array}{l}\infty \\
\bar{\sigma} \\
\dot{\sigma} \\
\dot{\sigma}\end{array}$ & $\begin{array}{l}\widehat{\infty} \\
0 \\
0 \\
1 \\
0 \\
\text { in } \\
\text { o } \\
\text { nn }\end{array}$ & & $\begin{array}{l}\bar{m} \\
\stackrel{1}{n} \\
\sim\end{array}$ & $\begin{array}{l}\tilde{E} \\
\tilde{E} \\
\tilde{\sigma}\end{array}$ & 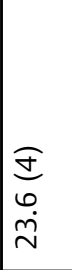 & 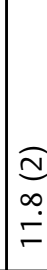 & 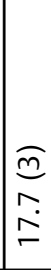 & \begin{tabular}{|l|}
$\widehat{\infty}$ \\
$\bar{\cdot}$ \\
$\dot{f}$ \\
\end{tabular} & $\begin{array}{l}\hat{I} \\
\tilde{E} \\
\dot{\tilde{\theta}}\end{array}$ & & 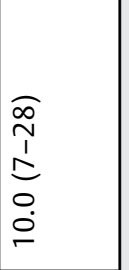 & & $\begin{array}{l}\text { ô } \\
\text { 足 } \\
\infty \\
\infty \\
\text { in }\end{array}$ & 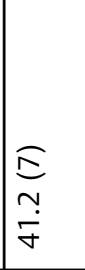 & $\begin{array}{l}E \\
\text { a } \\
\text { in }\end{array}$ & & 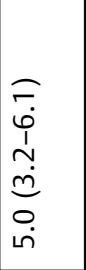 & 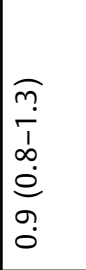 & 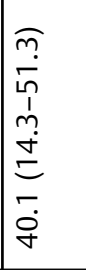 & 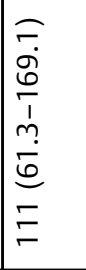 \\
\hline 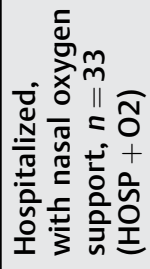 & $\begin{array}{l}\widehat{D} \\
\stackrel{D}{v} \\
0 \\
0 \\
0\end{array}$ & $\begin{array}{l}\hat{\infty} \\
\hat{1} \\
\hat{n} \\
\hat{\theta} \\
\hat{\sigma}\end{array}$ & & $\begin{array}{c}\tilde{m} \\
\stackrel{1}{d} \\
\stackrel{n}{\sim}\end{array}$ & $\begin{array}{l}\widehat{\widehat{N}} \\
\hat{\jmath} \\
\dot{0}\end{array}$ & $\begin{array}{c}0 \\
\underline{\sigma} \\
\infty \\
\infty \\
-\end{array}$ & $\begin{array}{l}\stackrel{\infty}{0} \\
\sim \\
\stackrel{\sim}{N}\end{array}$ & 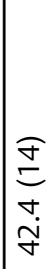 & $\begin{array}{l}\widehat{\Xi} \\
m \\
\dot{\omega}\end{array}$ & $\begin{array}{l}\stackrel{\infty}{\infty} \\
\stackrel{\infty}{\infty} \\
\infty \\
\infty \\
\infty\end{array}$ & & 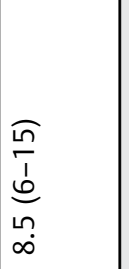 & & $\begin{array}{l}0 \\
0 \\
\circ \\
0\end{array}$ & $\mid \begin{array}{l}0 \\
0 \\
0 \\
0\end{array}$ & $\mid \begin{array}{l}\widehat{\Xi} \\
\bar{\sigma}\end{array}$ & & 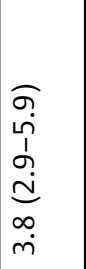 & 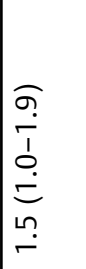 & $\begin{array}{l}0 \\
0 \\
0 \\
0 \\
1 \\
n \\
0 \\
\infty \\
\infty \\
\end{array}$ & 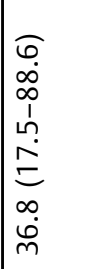 \\
\hline 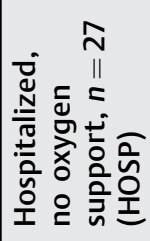 & 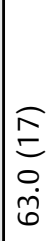 & 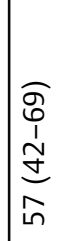 & & $\begin{array}{l}\frac{1}{n} \\
1 \\
\\
\sim\end{array}$ & $\begin{array}{c}\widehat{\tilde{m}} \\
\stackrel{n}{N} \\
\infty \\
\dot{\sigma} \\
\end{array}$ & 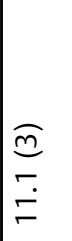 & 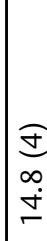 & 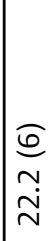 & 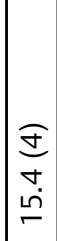 & 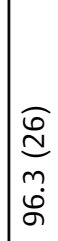 & & 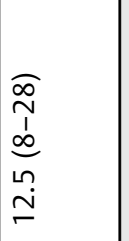 & & $\begin{array}{l}o \\
\text { o } \\
\stackrel{0}{0}\end{array}$ & $\begin{array}{l}\widehat{a} \\
0 \\
0 \\
0\end{array}$ & $\begin{array}{l}a \\
0 \\
0 \\
0\end{array}$ & & 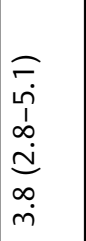 & 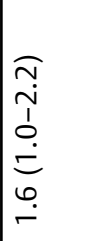 & 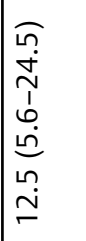 & 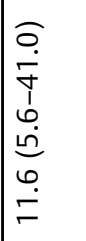 \\
\hline 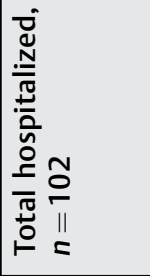 & 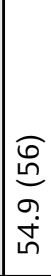 & 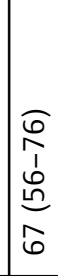 & & $\begin{array}{l}\tilde{F} \\
I \\
\stackrel{5}{N}\end{array}$ & $\begin{array}{l}\widehat{\sigma} \\
\emptyset \\
\tilde{\sigma} \\
\tilde{b}\end{array}$ & 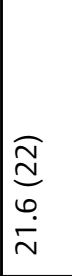 & 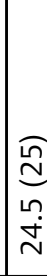 & $\begin{array}{l}\stackrel{\mathcal{F}}{m} \\
m \\
m \\
m\end{array}$ & 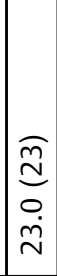 & $\begin{array}{l}\widehat{\infty} \\
\infty \\
m \\
\underline{\infty} \\
\dot{\infty}\end{array}$ & & $\begin{array}{l}\widehat{\sigma} \\
1 \\
\\
0 \\
\dot{\nabla}\end{array}$ & & 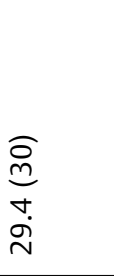 & $\begin{array}{l}\widehat{\sigma} \\
\infty \\
\infty \\
\infty\end{array}$ & 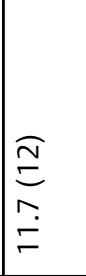 & $\widehat{\alpha}$ & 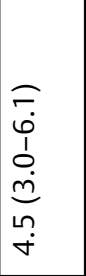 & 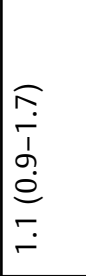 & 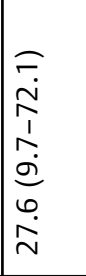 & 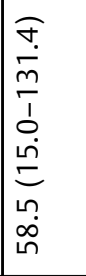 \\
\hline 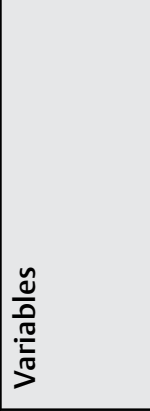 & 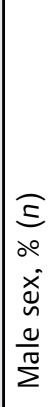 & 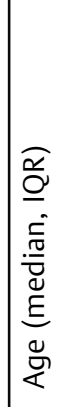 & 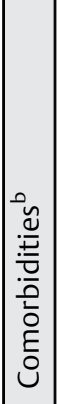 & 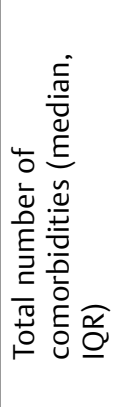 & 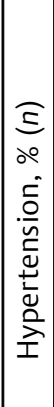 & 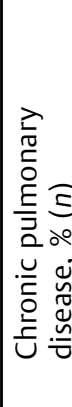 & 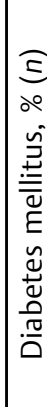 & 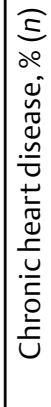 & 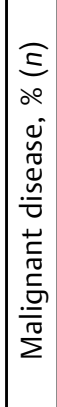 & 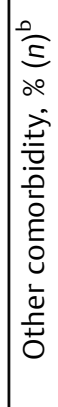 & 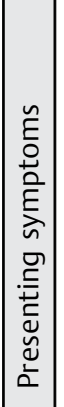 & 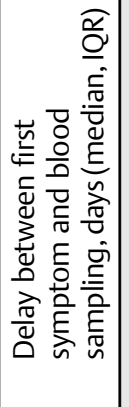 & 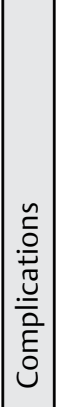 & 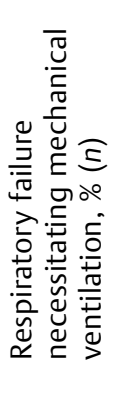 & 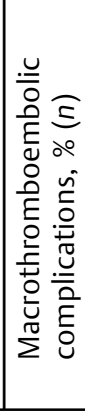 & 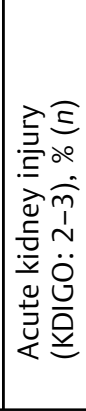 & 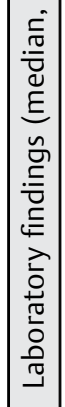 & 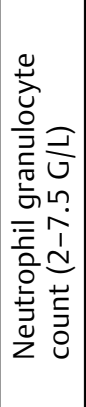 & 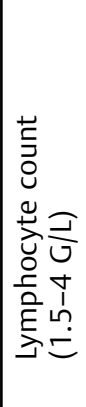 & 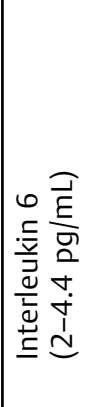 & 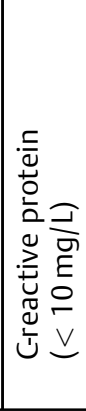 \\
\hline
\end{tabular}




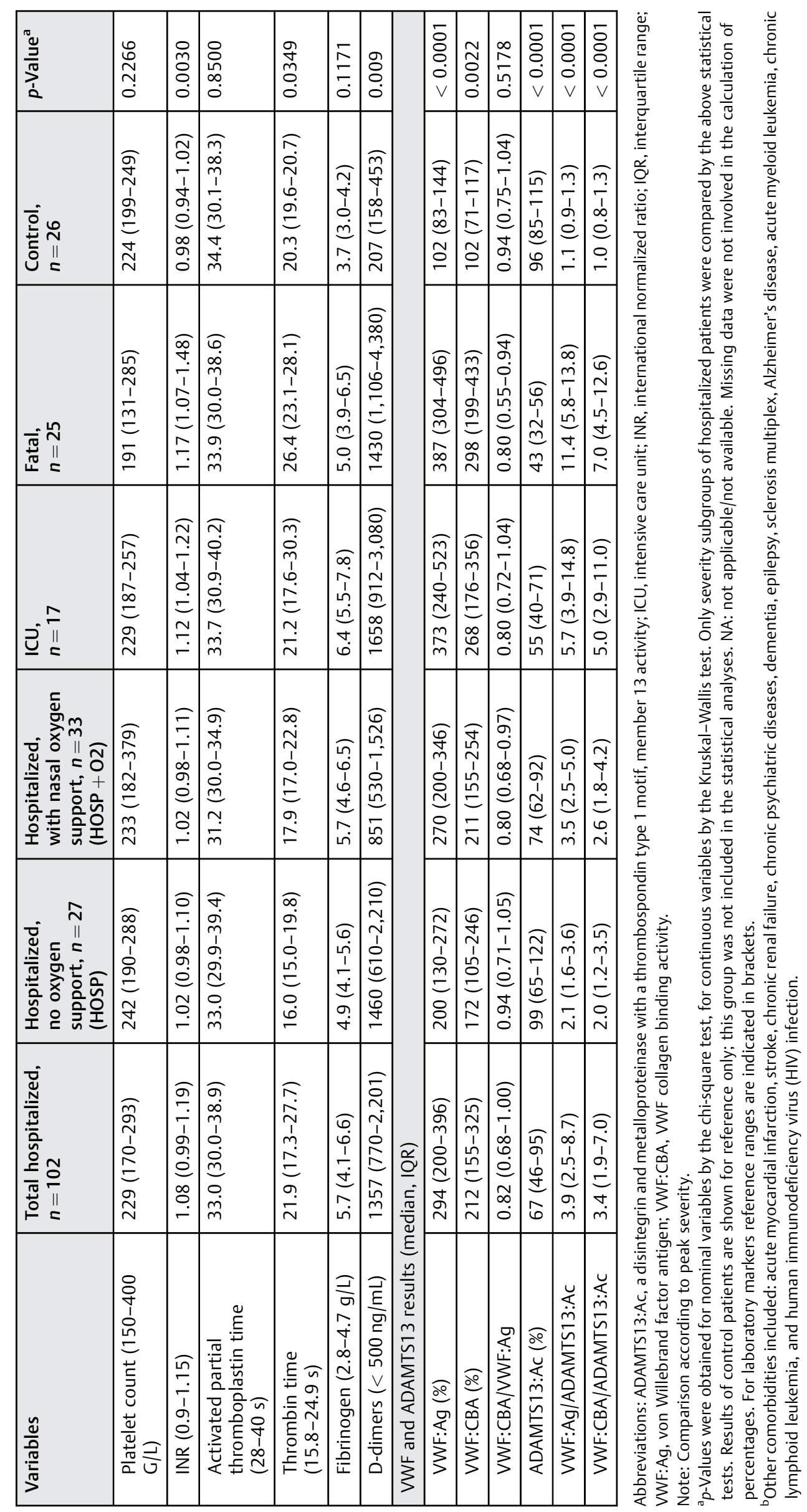




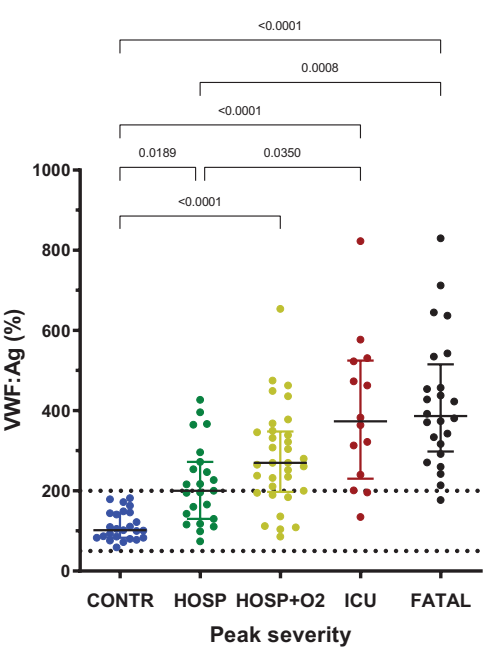

B

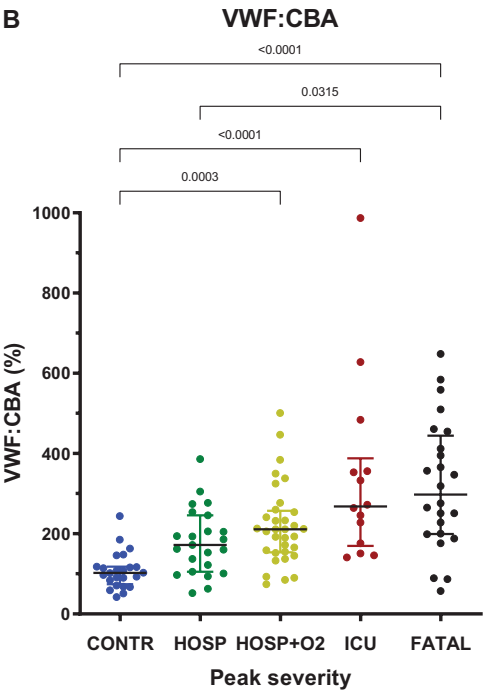

C

VWF:CBA / VWF:Ag

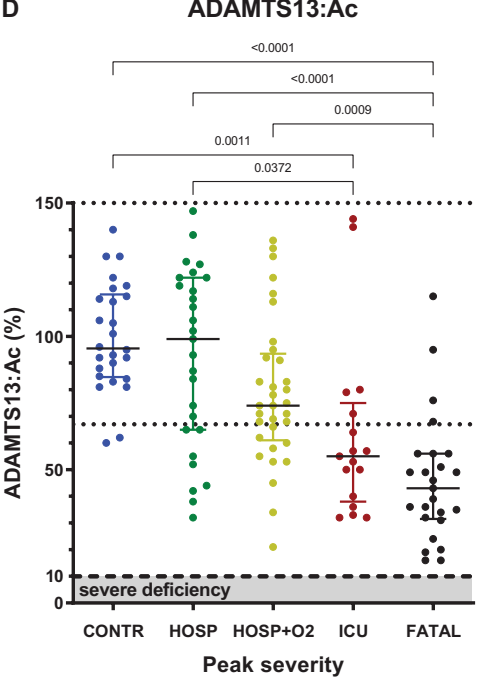

E
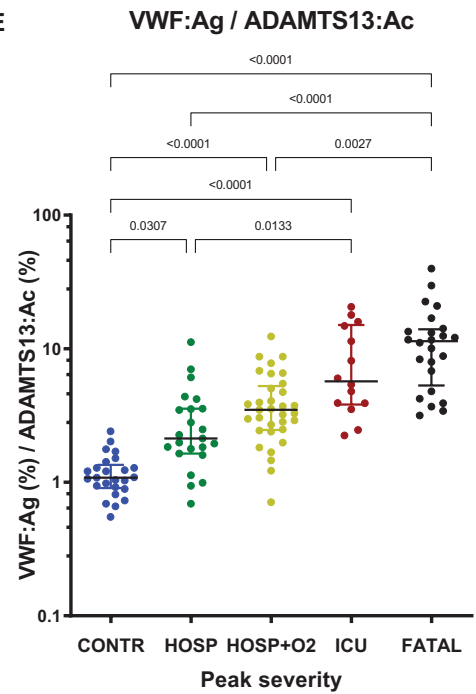

$\mathbf{F}$

VWF:CBA / ADAMTS13:Ac
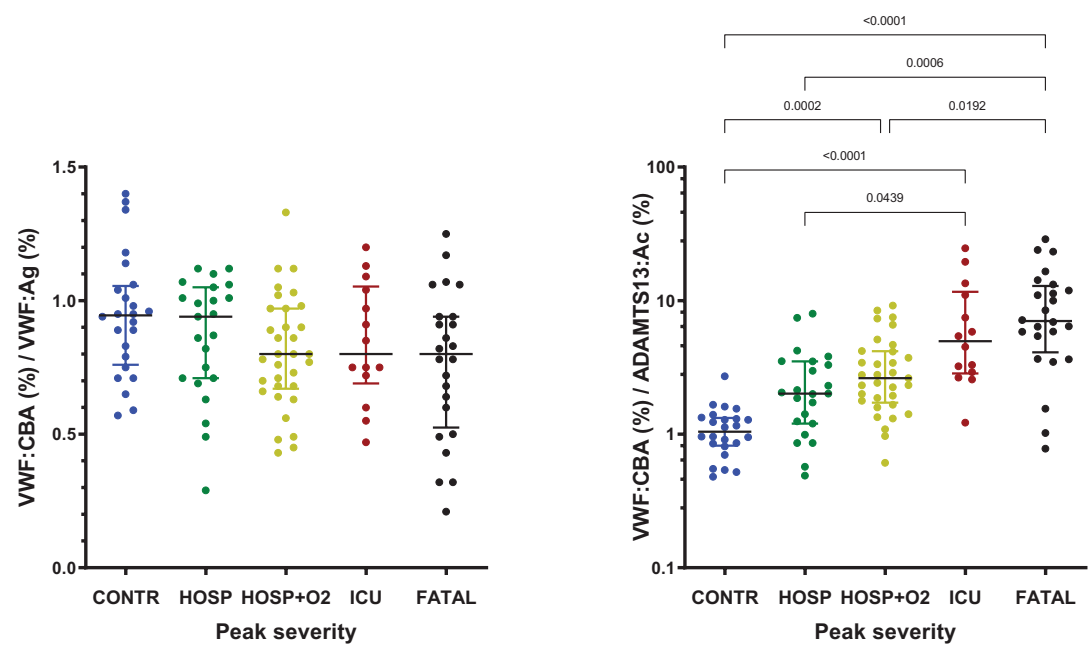

Fig. 1 von Willebrand factor (VWF) antigen (VWF:Ag), VWF collagen binding activity (VWF:CBA), a disintegrin and metalloproteinase with a thrombospondin type 1 motif, member 13 activity (ADAMTS13:AC), and their ratios in groups based on the peak severity of the COVID-19 disease. Median and interquartile ranges are plotted. The dotted lines indicate the upper and lower limits of the normal range; the gray area below the dashed line on panel $\mathbf{D}$ indicates severe ADAMTS13 deficiency. ( $p$-values of Dunn's multiple comparison tests below 0.05 are shown.) 
The levels of VWF:Ag and VWF:CBA showed a gradual increase in parallel with increased disease severity. Roughly half of the patients in the HOSP group had VWF:Ag levels above the upper limit of the reference range (200\%), whereas VWF:Ag concentrations were increased in almost all fatal cases. The VWF:CBA/VWF:Ag ratios in groups of hospitalized COVID-19 patients did not differ significantly from each other and from those of control subjects.

ADAMTS13:Ac markedly decreased in severe COVID-19 cases (FATAL and ICU groups), whereas it was normal or only slightly decreased in cases of moderate severity (HOSP and HOSP + O2). The proportion of patients with ADAMTS13:Ac levels below the lower limit of the reference range (67\%) was around $30 \%$ in moderate cases, $70.6 \%$ in the ICU group, and $84.0 \%$ in the FATAL group. It is important to note, however, that none of the hospitalized COVID-19 patients had severely deficient $(<10 \%)$ ADAMTS13:Ac values.

In consequence of the above changes, the VWF:Ag/ADAMTS13:Ac and VWF:CBA/ADAMTS13 ratios in- creased across groups in parallel with disease severity: the median VWF:Ag/ADAMTS13:Ac ratio was over five times higher in the FATAL group compared with the HOSP group.

\section{Associations of von Willebrand Factor Levels and ADAMTS13 Activity with Laboratory and Clinical Parameters}

The above parameters-VWF:Ag, VWF:CBA, ADAMTS13:Ac, and VWF:Ag/ADAMTS13:Ac ratio-correlated with several laboratory parameters associated with disease severity. These correlations are presented in detail in - Supplementary Table S2 (available in the online version). Briefly, VWF:Ag levels showed moderate positive correlations (Spearman's $r>0.3, p<0.01$ ) with markers of inflammation (CRP, procalcitonin, ferritin), urea, and lactate dehydrogenase. ADAMTS13:Ac inversely correlated (Spearman's $r<-0.3, p<0.01$ ) with the above parameters as well as with neutrophil granulocyte count, D-dimer, red blood cell distribution width, and IL-6 values. In addition, ADAMTS13:

Table 2 Laboratory data of mild (HOSP and HOSP + O2) and severe (ICU and FATAL) COVID-19 cases

\begin{tabular}{|c|c|c|c|}
\hline Variables & $\begin{array}{l}\text { Mild } \\
(\mathrm{HOSP} / \mathrm{HOSP}+02) \\
n=60\end{array}$ & $\begin{array}{l}\text { Severe } \\
\text { (ICU/FATAL) } \\
n=42\end{array}$ & $p$-Value ${ }^{a}$ \\
\hline Neutrophil granulocyte count $(2-7.5 \mathrm{G} / \mathrm{L})$ & $3.8(2.8-5.8)$ & $5.6(3.2-9.4)$ & 0.0022 \\
\hline Lymphocyte count (1.5-4 G/L) & $1.5(1.0-2.0)$ & $0.9(0.6-1.2)$ & $<0.0001$ \\
\hline Interleukin $6(2-4.4$ pg/mL) & $16.9(6.2-45.1)$ & $47.8(20.4-197.0)$ & 0.0001 \\
\hline C-reactive protein $(<10 \mathrm{mg} / \mathrm{L})$ & $24.1(8.4-73.5)$ & $123.9(54.9-195.4)$ & $<0.0001$ \\
\hline Platelet count (150-400 G/L) & $242(189-349)$ & $222(147-285)$ & 0.0602 \\
\hline INR (0.9-1.15) & $1.02(0.98-1.10)$ & $1.15(1.06-1.38)$ & 0.0002 \\
\hline Fibrinogen $(2.8-4.7 \mathrm{~g} / \mathrm{L})$ & $5.3(4.4-6.4)$ & $6.0(4.1-6.9)$ & 0.5234 \\
\hline D-dimers $(<500 \mathrm{ng} / \mathrm{mL})$ & $1,105(580-1,752)$ & $1,620(1,090-3,090)$ & 0.0024 \\
\hline \multicolumn{4}{|l|}{ Complement parameters } \\
\hline Classical pathway (48-103 CH50/mL) & $77(67-89)$ & $71(48-85)$ & 0.0678 \\
\hline Lectin pathway (35-125\%) & $73(6-141)$ & $56(6-134)$ & 0.7529 \\
\hline Alternative pathway (70-125\%) & $94(79-107)$ & $80(58-96)$ & 0.0038 \\
\hline C3 $(0.9-1.8 \mathrm{~g} / \mathrm{L})$ & $1.31(1.13-1.48)$ & $1.12(0.86-1.37)$ & 0.0050 \\
\hline $\mathrm{C} 4(0.15-0.55 \mathrm{~g} / \mathrm{L})$ & $0.37(0.29-0.46)$ & $0.29(0.21-0.51)$ & 0.1530 \\
\hline sC5b9 (110-252 ng/mL) & $268(192-372)$ & $364(242-529)$ & 0.0203 \\
\hline C3a (70-270 ng/mL) & $220(134-294)$ & $353(216-511)$ & 0.0001 \\
\hline C3a/C3 (ng/mg) & $154(113-225)$ & $316(186-565)$ & $<0.0001$ \\
\hline \multicolumn{4}{|l|}{ VWF and ADAMTS13 } \\
\hline VWF:Ag (50-200\%) & $242(175-335)$ & $382(292-523)$ & $<0.0001$ \\
\hline VWF:CBA (\%) & $193(141-250)$ & $274(199-412)$ & 0.0002 \\
\hline VWF:CBA/VWF:Ag & $0.84(0.69-1.00)$ & $0.80(0.60-0.97)$ & 0.6155 \\
\hline ADAMTS13:Ac (67-150\%) & $81(64-114)$ & $49(34-57)$ & $<0.0001$ \\
\hline VWF:Ag/ADAMTS13:Ac & $3.0(1.9-4.3)$ & $9.4(4.2-14.2)$ & $<0.0001$ \\
\hline VWF:CBA/ADAMTS13:Ac & $2.3(1.5-3.6)$ & $6.4(3.5-11.9)$ & $<0.0001$ \\
\hline
\end{tabular}

Abbreviations: ADAMTS13:Ac, a disintegrin and metalloproteinase with a thrombospondin type 1 motif, member 13 activity; HOSP, hospitalized, no oxygen support; HOSP + O2, hospitalized, with nasal oxygen support; ICU, intensive care unit; INR, international normalized ratio; VWF:Ag, von Willebrand factor antigen; VWF:CBA, VWF collagen binding activity.

${ }^{a} p$-Values of the Mann-Whitney $U$ test are shown. 

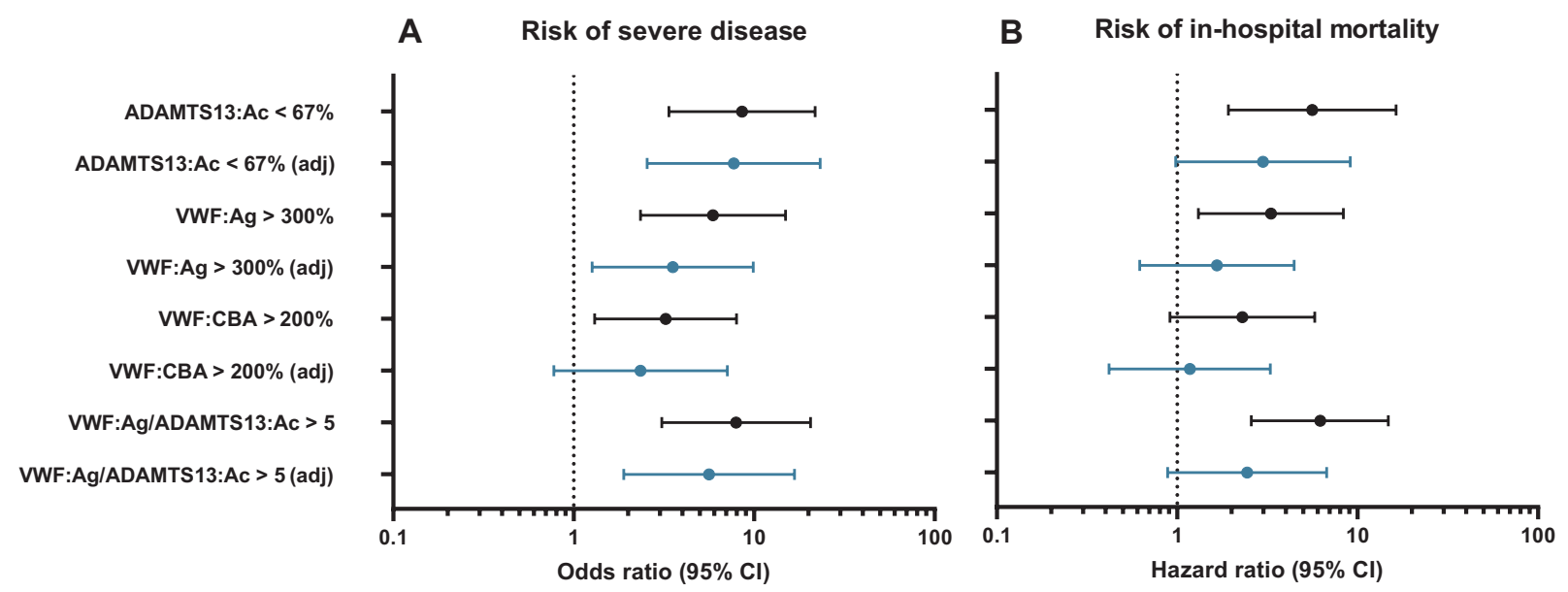

Fig. 2 Associations of low a disintegrin and metalloproteinase with a thrombospondin type 1 motif, member 13 activity (ADAMTS13:AC) and high von Willebrand factor (VWF) antigen (VWF:Ag) or VWF collagen binding activity (VWF:CBA) values with the risk of developing severe disease (A) and with the risk of in-hospital mortality (B). ADAMTS13:Ac values below 67\% were considered low, whereas VWF:Ag concentrations above $300 \%$, VWF:CBA values above 200\%, and VWF:Ag/ADAMTS13:Ac ratios above 5 were considered high. Fatal cases and cases requiring intensive care were regarded as severe. Odds ratios of logistic regression models (A), hazard ratios of Cox proportional hazard models (B), and their $95 \%$ confidence intervals $(95 \% \mathrm{Cls})$ are shown. Results of multivariable regression models in which each of the above variables were adjusted for a baseline model (adj) including age (in decades), number of comorbidities, and C-reactive protein (CRP) level (grouped according to median and quartiles) are shown in blue. (Results of the above logistic and Cox regression models are also presented as tables-in

- Supplementary Tables S4 and S5, respectively.)

Ac showed moderate positive correlations with the lymphocyte count, red blood cell count, and hemoglobin levels, with the activity of the complement alternative pathway and with the concentrations of its components and regulators ( $\mathrm{C} 3$, factor I, factor $\mathrm{H}$ ).

Interestingly, apart from the above described moderate inverse correlation between ADAMTS13:Ac and D-dimer level, neither ADAMTS13:Ac nor VWF:Ag or VWF:CBA correlated with other parameters of hemostasis and coagulation (platelet count, prothrombin time, activated partial thromboplastin time, thrombin time, or fibrinogen level).

ADAMTS13:Ac was lower, whereas VWF:CBA was higher in patients older than 67 years (median age in the cohort). Furthermore, ADAMTS13:Ac tended to be lower in patients with acute kidney injury (KDIGO 2 or 3 ), and was significantly lower in patients with malignant diseases (- Supplementary Table 53, available in the online version). After stratification according to disease severity and age or malignancy, we found that the differences in ADAMTS13:Ac, VWF:Ag, or VWF:CBA were not statistically significant in any subgroup (-Supplementary Figs. S2 and s3, available in the online version). There was no difference in the VWF:Ag, VWF:CBA, or ADAMTS13:Ac values between severe COVID-19 patients with and without macrothromboembolic complications.

\section{von Willebrand Factor Antigen, Collagen Binding Activity, and ADAMTS13 Activity as Biomarkers of Disease Severity}

To assess the potential of VWF:Ag, VWF:CBA, and ADAMTS13:Ac as biomarkers of COVID-19 disease severity, we divided the patients into two groups (in accordance with the WHO Ordinal Scale for Clinical Improvement): fatal cases and cases necessitating ICU admission were considered severe (ICU and FATAL groups, $n=42$ ), whereas other cases requiring hospitalization (HOSP and $\mathrm{HOSP}+\mathrm{O} 2$ groups, $n=60$ ) were considered of moderate severity. Laboratory results of mild and severe cases are summarized in - Table 2 .

Based on the median values of VWF:Ag (294\%), VWF:CBA (212\%), and ADAMTS13:Ac (67\%) in our cohort, we chose $300 \%$ as a cutoff value for VWF:Ag, $200 \%$ for VWF:CBA, and 67\% for ADAMTS13:Ac; the latter coincided with the lower limit of the ADAMTS13:Ac reference range. According to the results of ROC curve analysis, these cutoff values were almost optimal for distinguishing between moderate and severe COVID-19 cases (-Supplementary Fig. S4, available in the online version).

According to the results of logistic regression analysis, we found that patients with VWF:Ag above 300\%, VWF:CBA above $200 \%$, or ADAMTS13:Ac below 67\% were 5.91 (95\% confidence interval [CI]: 2.34-14.93), 3.23 (1.31-7.98), and 8.56 (3.37-21.73) times more likely to have severe COVID-19 disease, respectively, when compared with other patients (-Fig. 2A, - Supplementary Table S4, available in the online version). Importantly, VWF:Ag and ADAMTS13:Ac remained significant indicators of disease severity in multivariable models even after adjusting for a baseline model consisting of age, the number of comorbidities, and CRP concentrations. The VWF:Ag/ADAMTS13:Ac ratio was not superior to ADAMTS13:Ac alone in differentiating between severe and moderate COVID-19 cases.

\section{von Willebrand Factor Antigen, Collagen Binding Activity, and ADAMTS13 Activity as Predictors of In- Hospital Mortality}

Twenty-five COVID-19 patients in our study cohort died during the hospital stay, which means that the overall inhospital mortality was $24.5 \%$. Laboratory parameters of survivors and nonsurvivors are summarized in - Table 3. 
Table 3 Laboratory data of COVID-19 patients who later survived or deceased

\begin{tabular}{|c|c|c|c|}
\hline Variables & $\begin{array}{l}\text { Survived } \\
\text { (HOSP/HOSP }+02 / \mathrm{ICU}) \\
n=77\end{array}$ & $\begin{array}{l}\text { Deceased } \\
\text { (FATAL) } \\
n=25\end{array}$ & $p$-Value ${ }^{a}$ \\
\hline Neutrophil granulocyte count (2-7.5 G/L) & $3.9(2.9-5.9)$ & $6.0(4.2-10.3)$ & 0.0050 \\
\hline Lymphocyte count (1.5-4 G/L) & $1.4(0.9-1.9)$ & $0.8(0.5-1.1)$ & 0.0002 \\
\hline Interleukin 6 (2-4.4 pg/mL) & $19.0(6.9-48.7)$ & $90.4(34.6-267.3)$ & $<0.0001$ \\
\hline C-reactive protein $(<10 \mathrm{mg} / \mathrm{L})$ & $36.8(10.8-97.4)$ & $149.1(54.9-196.8)$ & 0.0002 \\
\hline Platelet count (150-400 G/L) & $237(188-306)$ & $194(131-285)$ & 0.0592 \\
\hline INR (0.9-1.15) & $1.05(0.98-1.14)$ & $1.17(1.07-1.48)$ & 0.0032 \\
\hline Fibrinogen $(2.8-4.7 \mathrm{~g} / \mathrm{L})$ & $5.7(4.6-6.8)$ & $5.0(3.9-6.5)$ & 0.2696 \\
\hline D-dimers (< $500 \mathrm{ng} / \mathrm{mL})$ & $1,140(610-1,900)$ & $1,430(1,106-4,380)$ & 0.0102 \\
\hline \multicolumn{4}{|l|}{ Complement parameters } \\
\hline Classical pathway (48-103 CH50/mL) & $74(66-89)$ & $63(44-80)$ & 0.0084 \\
\hline Lectin pathway (35-125\%) & $72(4-141)$ & $56(9-134)$ & 0.7513 \\
\hline Alternative pathway (70-125\%) & $94(80-103)$ & $60(35-87)$ & $<0.0001$ \\
\hline C3 $(0.9-1.8 \mathrm{~g} / \mathrm{L})$ & $1.31(1.11-1.49)$ & $1.05(0.66-1.20)$ & $<0.0001$ \\
\hline $\mathrm{C} 4(0.15-0.55 \mathrm{~g} / \mathrm{L})$ & $0.37(0.26-0.48)$ & $0.27(0.16-0.43)$ & 0.0468 \\
\hline sC5b9 (110-252 ng/mL) & $281(203-410)$ & $364(246-498)$ & 0.1288 \\
\hline C3a $(70-270 \mathrm{ng} / \mathrm{mL})$ & $237(141-337)$ & $375(196-459)$ & 0.0095 \\
\hline C3a/C3 (ng/mg) & $179(123-271)$ & $337(266-651)$ & $<0.0001$ \\
\hline \multicolumn{4}{|l|}{ VWF and ADAMTS13 } \\
\hline VWF:Ag (50-200\%) & $257(195-365)$ & $387(304-496)$ & 0.0002 \\
\hline VWF:CBA (\%) & $205(151-272)$ & $298(199-433)$ & 0.0058 \\
\hline VWF:CBA/VWF:Ag & $0.83(0.69-1.01)$ & $0.80(0.55-0.94)$ & 0.3812 \\
\hline ADAMTS13:Ac (67-150\%) & $74(55-106)$ & $43(32-56)$ & $<0.0001$ \\
\hline VWF:Ag/ADAMTS13:Ac & $3.5(2.1-5.5)$ & $11.4(5.8-13.8)$ & $<0.0001$ \\
\hline VWF:CBA/ADAMTS13:Ac & $2.7(1.7-4.2)$ & $7.0(4.5-12.6)$ & $<0.0001$ \\
\hline
\end{tabular}

Abbreviations: ADAMTS13:Ac, a disintegrin and metalloproteinase with a thrombospondin type 1 motif, member 13 activity; HOSP, hospitalized, no oxygen support; $\mathrm{HOSP}+\mathrm{O} 2$, hospitalized, with nasal oxygen support; ICU, intensive care unit; INR, international normalized ratio; VWF:Ag, von Willebrand factor antigen; VWF:CBA, VWF collagen binding activity.

${ }^{a} p$-Values of the Mann-Whitney $U$ test are shown.

ADAMTS13:Ac was significantly lower, whereas VWF:Ag and VWF:CBA were significantly higher in samples of patients who later deceased, compared with survivors.

The above results suggest that these parameters might prove to be useful biomarkers for predicting the inhospital mortality of hospitalized COVID-19 patients (-Supplementary Fig. S5, available in the online version).

Indeed, in-hospital mortality was higher in patients with ADAMTS13:Ac below 67\% (41.2\% vs. 7.8\%, $p<0.0001$ ) or with VWF:Ag levels above 300\% (39.1\% vs. $12.5 \%, p=0.004$ ), compared with other patients. The difference between patients with low and high VWF:CBA levels was not statistically significant. Kaplan-Meier curves showing cumulative survival in the above groups are shown in -Fig. 3.

Finally, we generated Cox proportional hazard models to analyze the effect of decreased $(<67 \%)$ ADAMTS13:Ac and elevated VWF:Ag ( $>300 \%$ ) and VWF:CBA (>200\%) levels on the in-hospital mortality of COVID-19 patients. The hazard ratio was 5.59 (95\% CI: 1.92-16.32) for decreased ADAMTS13:Ac and 3.31 (1.31-8.34) for increased VWF:Ag (-Fig. 2B, -Supplementary Table S5, available in the online version) in univariable models.

However, the increased VWF:Ag and decreased ADAMTS13:Ac levels did not prove to be significant independent predictors of in-hospital mortality after adjusting to the above described baseline model including age, the number of comorbidities, and CRP concentration. The VWF:CBA was not a significant predictor, whereas the VWF:Ag/ADAMTS13:Ac ratio was similar to ADAMTS13:Ac alone in predicting inhospital mortality.

\section{The Concomitant Presence of Decreased ADAMTS13 Activity and Increased Complement Activation as a Predictor of Severity and In-Hospital Mortality}

Previously we described that the level of C3a-marker of complement activation and anaphylatoxin-was increased, 
A

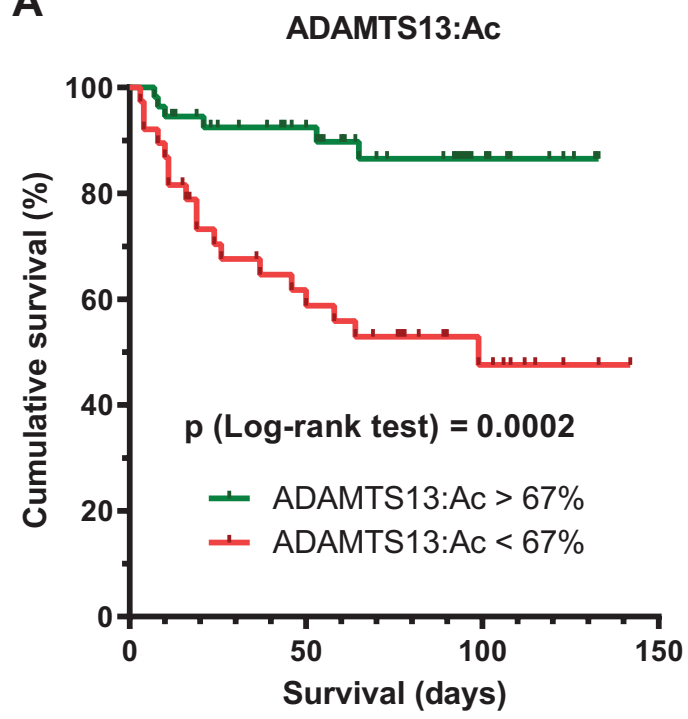

C

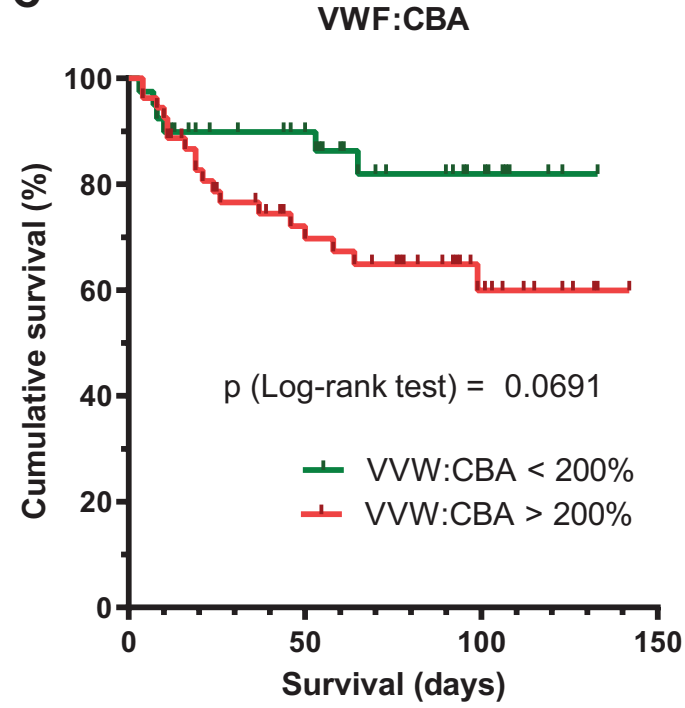

B
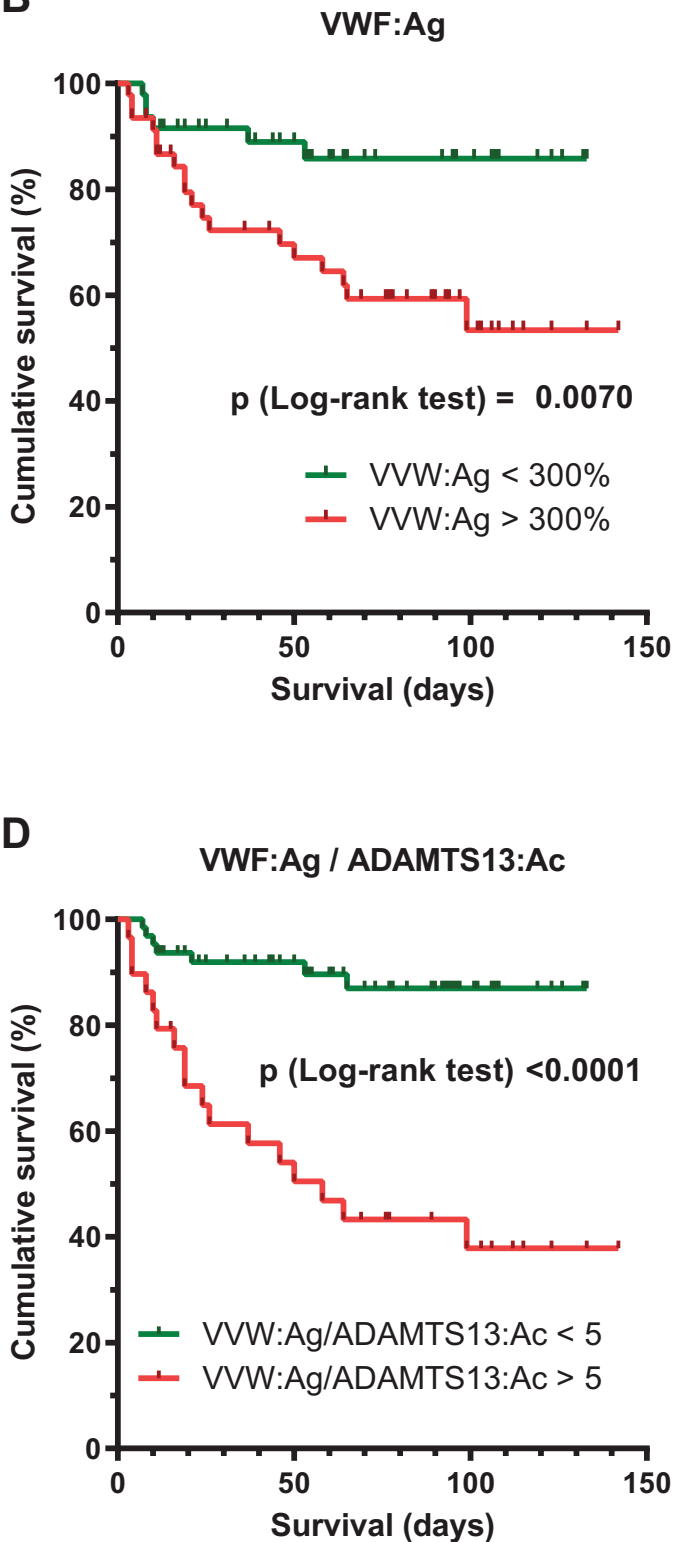

Fig. 3 Mortality in patients according to a disintegrin and metalloproteinase with a thrombospondin type 1 motif, member 13 activity (ADAMTS13:Ac), von Willebrand factor (VWF) antigen (VWF:Ag), VWF collagen binding activity (VWF:CBA), and VWF:Ag/ADAMTS13:Ac ratio. Kaplan-Meier curves (in-hospital mortality plotted against time from hospital admission to death or last follow-up) for patients above and below 67\% ADAMTS13:Ac (A), 300\% VWF:Ag (B), 200\% VWF:CBA (C), and a VWF:Ag/ADAMTS13:Ac ratio of 5 (D) are shown.

whereas the level of complement factor C3 was decreased in fatal COVID-19 cases. $^{29}$ We found that patients with a C3a/C3 ratio over $200 \mathrm{ng} / \mathrm{mg}$-indicating complement overactivation and consumption-had a higher risk of death compared with other patients. Along these lines, we investigated whether there is a relationship between ADAMTS13:Ac, complement overactivation and consumption, and the severity and outcome of COVID-19. Accordingly, we applied stratified multivariable statistical analyses with interaction terms. Hospitalized patients were divided into four subgroups based upon their ADAMTS13:Ac and
C3a/C3 ratio. The subgroups are described in detail in -Table 4.

Peak disease severity according to ADAMTS13:Ac and $\mathrm{C} 3 \mathrm{a} / \mathrm{C} 3$ values are shown in - Fig. 4A. Respiratory failure requiring intubation and mechanical ventilation was more frequent in the group of patients who had low ADAMTS13:Ac and high $\mathrm{C} 3 \mathrm{a} / \mathrm{C} 3$ ratio in comparison with the other groups ( $70.4 \%$ vs. $6.9 \%, 18.4 \%$, and $13.6 \%$, odds ratio $>10$ and $p<0.0004$ for each comparison).

In-hospital mortality was also considerably higher in the former subgroup than in any other subgroup (66.7\% vs. $6.9 \%$, 
Table 4 Characteristics of hospitalized COVID-19 patients in subgroups with different combinations of normal (>67\%) or low $(<67 \%)$ ADAMTS13:Ac and low $(<200 \mathrm{ng} / \mathrm{mg})$ or high $(>200 \mathrm{ng} / \mathrm{mg})$ complement C3a/C3 ratio

\begin{tabular}{|c|c|c|c|c|c|}
\hline Variables & $\begin{array}{l}\text { ADAMTS13:AC } \\
\text { normal, } \\
\text { C3a/C3 low } \\
n=29\end{array}$ & $\begin{array}{l}\text { ADAMTS13:AC } \\
\text { normal, } \\
\text { C3a/C3 high } \\
n=22\end{array}$ & $\begin{array}{l}\text { ADAMTS13:Ac low, } \\
\text { C3a/C3 low } \\
n=22\end{array}$ & $\begin{array}{l}\text { ADAMTS13:Ac low, } \\
\text { C3a/C3 high } \\
n=27\end{array}$ & $p$-Value ${ }^{a}$ \\
\hline Male sex, \% (n) & $62.1(18)$ & $50.0(11)$ & $59.1(13)$ & $48.1(13)$ & 0.690 \\
\hline Age (median, IQR) & $57(40-66)$ & $67(58-74)$ & $66(54-70)$ & $76(69-79)$ & $<0.001$ \\
\hline \multicolumn{6}{|l|}{ Comorbidities } \\
\hline $\begin{array}{l}\text { Total number of } \\
\text { comorbidities (median, IQR) }\end{array}$ & $2(1-3)$ & $2.5(1-3)$ & $2(1-3)$ & $3(2-5)$ & 0.042 \\
\hline Hypertension, \% (n) & $58.6(17)$ & $63.6(14)$ & $59.1(13)$ & $74.1(20)$ & 0.618 \\
\hline $\begin{array}{l}\text { Chronic pulmonary } \\
\text { disease, \% (n) }\end{array}$ & $13.8(4)$ & $31.8(7)$ & $22.7(5)$ & $22.2(6)$ & 0.497 \\
\hline Diabetes mellitus, \% (n) & $20.7(6)$ & $27.3(6)$ & $13.6(3)$ & $33.3(9)$ & 0.409 \\
\hline Chronic heart disease, \% ( $n$ ) & $31.0(9)$ & $27.3(6)$ & $22.7(5)$ & $48.1(13)$ & 0.238 \\
\hline Malignant disease, \% (n) & $3.4(1)$ & $9.1(2)$ & $31.8(7)$ & $48.1(13)$ & $<0.001$ \\
\hline \multicolumn{6}{|l|}{ Presenting symptoms } \\
\hline $\begin{array}{l}\text { Delay between first } \\
\text { symptom and sampling, days } \\
\text { (median, IQR) }\end{array}$ & $12(6-25)$ & $8(5-19)$ & $9(5-14)$ & $10(4-27)$ & 0.858 \\
\hline \multicolumn{6}{|l|}{ Complications } \\
\hline $\begin{array}{l}\text { Respiratory failure } \\
\text { necessitating mechanical } \\
\text { ventilation, \% (n) }\end{array}$ & $6.9(2)$ & $18.2(4)$ & $13.6(3)$ & $70.4(19)$ & $<0.001$ \\
\hline $\begin{array}{l}\text { Macrothromboembolic } \\
\text { complications, \% (n) }\end{array}$ & $0.0(0)$ & $18.2(4)$ & $4.5(1)$ & $7.4(2)$ & 0.085 \\
\hline $\begin{array}{l}\text { Acute kidney injury (KDIGO: } \\
2-3), \%(n)\end{array}$ & $3.4(1)$ & $13.6(3)$ & $4.5(1)$ & $25.9(7)$ & 0.042 \\
\hline Transfer to ICU, \% (n) & $6.9(2)$ & $27.3(6)$ & $31.8(7)$ & $77.8(21)$ & $<0.001$ \\
\hline Death, \% (n) & $6.9(2)$ & $9.1(2)$ & $9.1(2)$ & $66.7(18)$ & $<0.001$ \\
\hline \multicolumn{6}{|l|}{ Laboratory findings (median, IQR) } \\
\hline $\begin{array}{l}\text { Neutrophil granulocyte } \\
\text { count }(2-7.5 \mathrm{G} / \mathrm{L})\end{array}$ & $3.5(2.8-4.5)$ & $4.3(2.8-6.1)$ & $4.6(3.2-5.9)$ & $6.0(4.2-10.4)$ & 0.007 \\
\hline Lymphocyte count (1.5-4 G/L) & $1.8(1.0-2.1)$ & $1.1(0.9-1.7)$ & $1.0(0.9-1.5)$ & $1.0(0.7-1.4)$ & 0.008 \\
\hline Interleukin 6 (2-4.4 pg/mL) & $12.5(6.0-41.2)$ & $24.5(12.8-72.2)$ & $29.1(19.0-50.3)$ & $50.0(14.0-265.0)$ & 0.040 \\
\hline $\begin{array}{l}\text { C-reactive protein } \\
(<10 \mathrm{mg} / \mathrm{L})\end{array}$ & $15(6-41)$ & $77(30-145)$ & $45(14-108)$ & $149(42-195)$ & $<0.001$ \\
\hline Platelet count (150-400 G/L) & $233(192-282)$ & $236(129-388)$ & $236(173-348)$ & $204(163-285)$ & 0.545 \\
\hline INR (0.9-1.15) & $1.05(0.98-1.11)$ & $1.02(0.98-1.20)$ & $1.06(0.98-1.15)$ & $1.12(1.06-1.47)$ & 0.142 \\
\hline Fibrinogen $(2.8-4.7 \mathrm{~g} / \mathrm{L})$ & $5.1(4.2-6.6)$ & $5.0(4.0-6.6)$ & $5.8(4.9-7.6)$ & $5.7(4.4-6.5)$ & 0.700 \\
\hline D-dimers (< $500 \mathrm{ng} / \mathrm{mL})$ & $1,030(530-1,850)$ & $1,547(512-1,996)$ & $1,480(879-3,090)$ & $1,366(1,079-3,398)$ & 0.164 \\
\hline VWF:Ag, \% (50-200\%) & $247(160-332)$ & $266(222-317)$ & $240(136-396)$ & $392(292-543)$ & $<0.001$ \\
\hline VWF:CBA, \% & $192(137-233)$ & $199(146-241)$ & $193(145-338)$ & $332(200-461)$ & $<0.001$ \\
\hline VWF:CBA / VWF:Ag & $0.86(0.71-1.01)$ & $0.70(0.55-0.88)$ & $0.95(0.75-1.06)$ & $0.82(0.68-0.99)$ & 0.052 \\
\hline
\end{tabular}

Abbreviations: ADAMTS13:Ac, a disintegrin and metalloproteinase with a thrombospondin type 1 motif, member 13 activity; ICU, intensive care unit; INR, international normalized ratio; IQR, interquartile range; VWF:Ag, von Willebrand factor antigen; VWF:CBA, VWF collagen binding activity. Note: Two patients had missing C3a data; these patients were not included in any of the subgroups. Other comorbidities included are listed below $\mathbf{- T a b l e ~ 1 . ~ R e f e r e n c e ~ r a n g e s ~ o f ~ l a b o r a t o r y ~ m a r k e r s ~ a r e ~ i n d i c a t e d ~ i n ~ b r a c k e t s . ~}$

${ }^{a} p$-Values were obtained by the chi-square test for nominal variables, and by the Kruskal-Wallis test for continuous variables. 
A

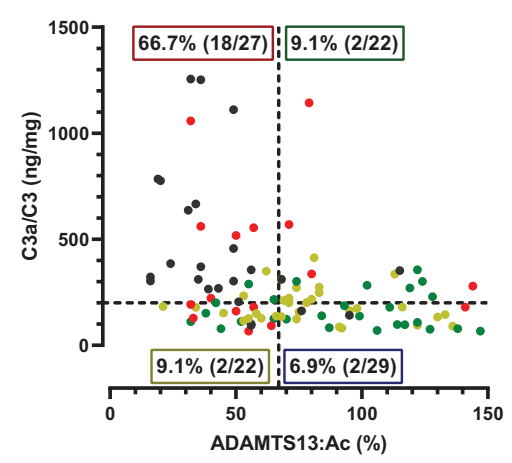

Peak severity

- FATAL (WHO 8)

ICU (WHO 6-7)

HOSP+O2 $2(\mathrm{WHO} 4)$

- $\operatorname{HOSP}(\mathrm{WHO} 3)$

B

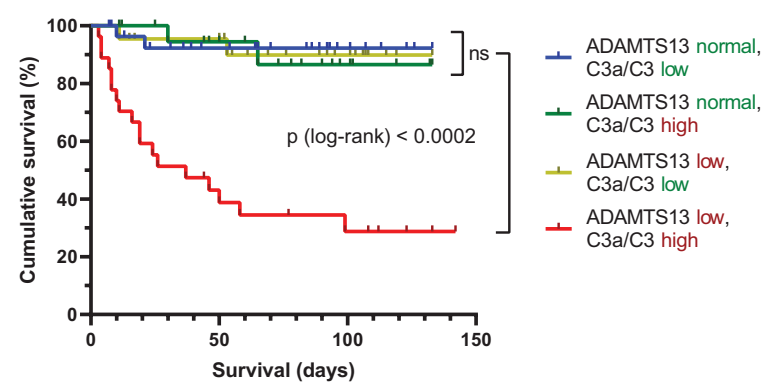

C

Risk of in-hospital mortality (ADAMTS13:Ac, C3a/C3 and their interaction)

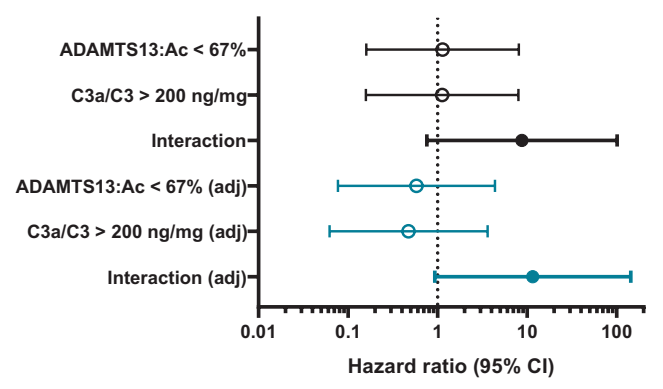

Fig. 4 Peak disease severity and in-hospital mortality in patients with different combinations of a disintegrin and metalloproteinase with a thrombospondin type 1 motif, member 13 activity (ADAMTS13:Ac) and $\mathrm{C} 3 \mathrm{a} / \mathrm{C} 3$ ratio, a marker of complement activation and

consumption. Peak disease severity according to ADAMTS13:Ac and $\mathrm{C} 3 \mathrm{a} / \mathrm{C} 3$ ratio is shown on $(\mathrm{A})$. Lines indicate the median-based cutoff values of ADAMTS13:Ac (67\%) and C3a/C3 (200 ng/mg) that were used to define subgroups with low or high values. High (normal) ADAMTS13:Ac and low C3a/C3 ratio are regarded as physiological, whereas low ADAMTS13:Ac and high C3a/C3 ratio are considered pathological. The proportions of deceased patients in each quartile are indicated in text boxes. (B) Kaplan-Meier curves (in-hospital mortality plotted against time) in the four subgroups. The survival in the low ADAMTS13:Ac and high C3a/C3 subgroup is significantly different from those of other subgroups $(p<0.0001, p=0.0001$, and $p<0.0001$, by pairwise log-rank comparisons), whereas it did not differ between the other three subgroups $(p=0.8369, p=0.8865$, and $p=0.9052$ ). Colors of the curves match those of text boxes on panel A. (C) Results of multivariable Cox proportional hazard ratio models composed of low ADAMTS13:Ac, high C3a/C3 ratio, and their statistical interaction. The model was adjusted to the baseline model composed of age, the number of comorbidities, and the $\mathrm{C}$ reactive protein (CRP) level; results of the adjusted model are shown in blue.
9.1\%, and 9.1\%; odds ratio $>19$ and $p<0.0001$ for each comparison).

In contrast, isolated low ADAMTS13:Ac or elevated C3a/C3 ratio, alone, were not associated with increased risk of respiratory failure or death.

Kaplan-Meier curves presented in - Fig. 4B show that the cumulative survival in patients with low ADAMTS13:Ac and high $\mathrm{C} 3 \mathrm{a} / \mathrm{C} 3$ ratio is clearly distinct from those of all other groups.

These results indicate that there is a statistical interaction between the above parameters: low ADAMTS13:Ac increases the risk of in-hospital mortality only in the setting of a high $\mathrm{C} 3 \mathrm{a} / \mathrm{C} 3$ ratio. To test how adjusting for our baseline model (consisting of age, number of comorbidities, and CRP level) influences the above statistical interaction, we prepared multivariable Cox proportional hazard models with interaction terms (presented in -Fig. $\mathbf{4 C}$ and -Supplementary Table S6, available in the online version). Our results demonstrate that adjusting for our baseline model did not affect the association of the statistical interaction between low ADAMTS13:Ac and high C3a/C3 ratio with in-hospital mortality.

\section{Discussion}

Our study provides the first observational evidence that the concomitant presence of decreased ADAMTS13:Ac and increased markers of complement activation is associated with COVID-19 severity and mortality. These results suggest that a potential interaction between the VWF-ADAMTS13 axis and complement activation may be a key factor in the pathogenesis of COVID-19.

First, to investigate the role of the VWF-ADAMTS13 axis in the pathogenesis of COVID-19, we measured ADAMTS13:Ac, VWF:Ag, and VWF:CBA levels in a cohort of 102 hospitalized COVID-19 patients of various disease severity and in a control group of 26 convalescent plasma donors.

We found that VWF:Ag and VWF:CBA levels were elevated in all groups of hospitalized COVID-19 patients; there was a continuous increase in these parameters in parallel with increasing COVID-19 severity. ADAMTS13:Ac, on the other hand, decreased in parallel with disease severity; most patients with severe COVID-19 (i.e., those who deceased or required intensive care) had ADAMTS13:Ac values below the lower limit of the normal range (67\%). As a consequence of the above alterations, the VWF:Ag/ADAMTS13:Ac ratioindicating the functional state of the VWF-ADAMTS13 axis -increased considerably, exceeding 10 in the group of nonsurvivors. The VWF:CBA/VWF:Ag ratio was variable, and did not differ significantly between groups based on disease severity. Severe ADAMTS13 deficiency was not observed in our cohort, in contrast to cases of thrombotic thrombocytopenic purpura patients with concomitant COVID-19 disease. $^{33}$

As ADAMTS13:Ac was significantly lower and VWF:Ag and VWF:CBA were significantly higher in severe COVID-19 
cases and in nonsurvivors than in moderate cases and in survivors, respectively, we assessed the potential of the above parameters as biomarkers of severity and as predictors of in-hospital mortality in hospitalized COVID-19 patients.

We found that patients with VWF:Ag levels over 300\% and those with ADAMTS13:Ac below the lower limit of normal (67\%) were 5.91 and 8.56 times more likely to have severe COVID-19 disease, whereas the risk of in-hospital mortality was 3.31 and 5.59 times higher in these groups, respectively. When adjusting for a baseline model composed of key clinical and laboratory parameters associated with the severity or mortality of COVID-19-age, number of comorbidities, and CRP concentration-decreased ADAMTS13:Ac and elevated VWF: Ag level remained significant predictors of disease severity, but were no longer significant predictors of in-hospital mortality.

Our results regarding the elevated VWF:Ag concentrations and the moderately decreased-but not deficient-ADAMTS13: Ac are in line with results described in other cohorts of hospitalized COVID-19 patients. ${ }^{11,12,14-16,19-21,34-39}$ The observations that the increase of VWF:Ag and the decrease of ADAMTS13:Ac were more pronounced in severe/critical COVID-19 than in moderate cases, and that elevated VWF:Ag and reduced ADAMTS13:Ac are thus predictors of in-hospital mortality in COVID-19, are also in agreement with results of previous studies. ${ }^{11,14-16,18-21,37-39}$

Taken together, our results support that the VWFADAMTS13 axis is involved in the pathogenesis of the COVID-19 disease. The hypoxic and inflammatory state characteristic for severe COVID-19 can increase the secretion and interfere with the cleavage of VWF by multiple mechanisms. $^{40,41}$ In particular, there is emerging evidence supporting the role of neutrophil granulocyte activation and the release of neutrophil extracellular traps (NETosis) in the pathogenesis of COVID-19 ${ }^{42,43}$; these processes may also affect the VWF-ADAMTS13 axis through the oxidative modification, sialylation or citrullination of its components, or by otherwise interfering with their interaction. ${ }^{44-48}$ If their cleavage is hindered by the above mechanisms, persisting ultra-large VWF multimers form large strings that are capable of binding platelets firmly. ${ }^{49}$

However, the ultra-large VWF multimers provide an ideal surface not only for platelet adhesion, but also for complement activation. ${ }^{50}$ Complement deposition in lung capillaries, ${ }^{51}$ and increased plasma levels of complement activation products support the activated state of the complement system in COVID-19. ${ }^{27,29,52}$ The concentrations of the activation products were found to be higher in severe COVID-19 patients, ${ }^{27,29,52}$ indicating that excessive complement activation is more likely in these cases. Furthermore, levels of complement activation products correlated with those of VWF and other markers of endothelial perturbation, ${ }^{52}$ supporting that there is a link between endothelial VWF secretion and complement activation.

Complement activation on the surface of endothelial cellbound ultra-large VWF multimers ${ }^{50}$ or exposure to complement activation products-C3a, C5a, C5b-9-induce prothrombotic and proinflammatory changes in endothelial cells, also termed as endothelial dysfunction. ${ }^{53-55}$ The con- sequentially increased release of VWF and the decreased expression of thrombomodulin further enhance complement activation and endothelial dysfunction. ${ }^{56}$ In addition to this direct positive feedback loop, there is another one involving platelets and neutrophil granulocytes. Complement activation products are able to activate platelets, neutrophil granulocytes, and macrophages. ${ }^{57}$ Platelet-decorated VWF strings provide an ideal scaffold for the adhesion of neutrophil granulocytes. ${ }^{58}$ If the neutrophils are preactivated, this may be followed by NETosis, which in turn induces tissue factor expression and thus augments the thrombotic potential of endothelial cells. ${ }^{59}$

In conclusion, if ultra-large VWF molecules are not cleaved upon release, the endothelial VWF secretion and complement activation amplify each other, eventually leading to immunothrombosis, a major cause of mortality in COVID-19. ${ }^{60}$ ADAMTS13, however, is able to break this vicious circle by cleaving the highly adhesive ultra-large VWF multimers.

Based on the above, we hypothesized, that the decrease of ADAMTS13:Ac would be more detrimental in the case of excessive complement activation-providing positive feedback in the above described ways-than in a setting of a wellregulated complement system.

To test this hypothesis, we compared disease outcomes in groups of hospitalized COVID-19 patients with different combinations of normal or decreased ADAMTS13:Ac and low or high levels of $\mathrm{C} 3 \mathrm{a} / \mathrm{C} 3$ ratios.

The $\mathrm{C} 3 \mathrm{a} / \mathrm{C} 3$ ratio was introduced in our previous analysis of the same cohort ${ }^{29}$ as a general marker of complement overactivation and consumption. Complement C3 is the central molecule of the complement system: all-the classical, lectin, and alternative-activation pathways converge on the level of C3. Upon its activation, the soluble C3a fragment is released, which is therefore a good indicator of complement activation. However, the absolute concentration of $\mathrm{C} 3 \mathrm{a}$ is dependent on the concentration of available C3 molecules. C3 concentrations, in turn, were found to moderately increase in parallel with disease severity-probably in consequence of the acute phase reaction-and then suddenly drop in fatal cases, due to complement consumption. ${ }^{29}$ Based on the above, we hypothesized that the $\mathrm{C} 3 \mathrm{a} / \mathrm{C} 3$ ratio better reflected the activated state of the complement system than C3a concentration alone. In line with this hypothesis, the $\mathrm{C} 3 \mathrm{a} / \mathrm{C} 3$ ratio proved to be a stronger predictor of inhospital mortality of COVID-19 patients in comparison to C3a in our previous study.

Most importantly, when we compared groups with different ADAMTS13:Ac and C3a/C3 ratios, we found that the frequency of respiratory failure and in-hospital death was indeed markedly higher in the group of patients who had decreased ADAMTS13:Ac and signs of excessive complement activation at the same time, whereas decreased ADAMTS13: Ac or increased complement activation alone were not found to be associated with increased disease severity or mortality. Adjusting to our baseline model did not influence the above described association between in-hospital mortality and the combination of low ADAMTS13:Ac and high C3a/C3 ratio. 
Interestingly, VWF:Ag concentration and VWF:CBA were also significantly higher in the group of patients with both low ADAMTS13:Ac and high C3a/C3 ratio, whereas it did not differ between the other subgroups ( $\rightarrow$ Table 4 ). This result supports that endothelial activation and increased VWF secretion might be a key link between decreased ADAMTS13:Ac, complement overactivation, and the severity of COVID-19.

The main strengths of our cohort were the concurrent determination of VWF:Ag, ADAMTS13:Ac, and the detailed characterization of the complement profile, which allowed us to investigate the interactions of the VWF-ADAMTS13 axis and complement activation. Our cohort, as a whole, represented a broad spectrum of COVID-19 severity, which, however, was divided into multiple, relatively homogenous subgroups. This enabled a detailed analysis of associations between COVID-19 severity and different laboratory parameters. As follow-up was complete in all cases, we were able to formally evaluate mortality in survival models. All relevant clinical and laboratory data were collected, which enabled us to adjust for the most important confounders.

A potential limitation of our study is its relatively small sample size of 102 patients. However, the subgroups based upon disease severity were nearly equal, which allowed us to perform reliable statistical analyses. Forming groups based on multiple variables, however, leads to subgroups with low numbers of individuals; results of statistical analyses have to be interpreted with caution in these cases.

A further limitation of our study was the high proportion of patients with malignant diseases, especially among cases with severe disease. However, there were no significant differences in ADAMTS13 or VWF levels between patients with and without malignant diseases in any severity subgroup. Thus, the lower ADAMTS13:Ac and higher VWF:Ag and VWF:CBA values observed in groups of higher severity are not attributable to the higher proportion of patients with malignant diseases in these groups. Accordingly, adjusting our models for the presence or absence of malignant diseases did not influence our results.

Furthermore, the median age was lower in control subjects and was higher in patients who subsequently died due to COVID-19 disease (FATAL group) compared with other groups. However, stratified analyses by disease severity showed no significant differences between patients below and above 67 years in any given subgroup. Furthermore, we adjusted all our models of severity or survival for a baseline model consisting of age, number of comorbidities, and CRP level.

Finally, it has to be noted that data on anticoagulation were not collected for all patients, although such treatment may have influenced the laboratory values of coagulation.

To conclude, in this study we have shown that the concurrent presence of decreased ADAMTS13:Ac and increased complement activation is associated with increased in-hospital mortality in COVID-19 patients. These results suggest that an interaction between the VWF-ADAMTS13 axis and complement system plays an important role in the patho- genesis of severe COVID-19 disease, most probably via triggering immunothrombosis. The specific molecular background of the above interaction has yet to be investigated. Importantly, our results indicate that if either ADAMTS13:Ac is normal or pathological complement activation is absent, the risk of in-hospital mortality is significantly lower in COVID-19. This finding raises the possibility of ADAMTS13 replacement therapy in selected cases with low ADAMTS13: Ac, and underlines the importance of studies on complement inhibitory drugs in COVID-19.

\section{What is known about this topic?}

- In a subset of patients infected with the SARS-CoV-2 virus, immunothrombosis develops in lung microvessels, which is a major cause of respiratory failure and mortality in COVID-19.

- Endothelial perturbation-which is a central event in the development of immunothrombosis-results in elevated VWF antigen concentrations, whereas ADAMTS13 activity is moderately decreased in COVID-19 patients.

- The complement system is also activated in COVID-19, levels of complement activation markers correlated with that of VWF and other markers of endothelial activation.

\section{What does this paper add?}

- In this study, we validated the role of increased VWF antigen concentrations and decreased ADAMTS13 activity as good markers of severity and predictors of inhospital mortality, and we report for the first time that concomitant changes in the VWF-ADAMTS13 axis and complement activation are associated with the severity and mortality of the COVID-19 disease.

- The risk of respiratory failure and of in-hospital mortality is higher in COVID-19 patients with concurrently decreased ADAMTS13 activity and increased C3a/C3 ratio-indicating complement overactivation and consumption-whereas decreased ADAMTS13 activity or high C3a/C3 ratio, alone, were not associated with increased risk of respiratory failure or death.

\section{Author Contributions}

G.S., B.M., D.C., L.H., E.K., L.C., and P.K. designed and performed laboratory determinations, interpreted data, and drafted the manuscript. G.S., Z.Z.P., and Z.P. conceptualized research, collected and analyzed clinical information and laboratory data, conducted statistical analysis, interpreted data, and wrote the manuscript. M. R., V.M., Z.F., Z.I., J.G., L.G., P.R., B.S., B.L., J.S., I.B., T.M., I.V.-N. took part in the conceptualization, collected and analyzed clinical information, interpreted and supervised data, and drafted the manuscript. All authors critically revised the final manuscript. 


\section{Funding}

The research was financed by the Higher Education Institutional Excellence Programme of the Ministry of Human Capacities in Hungary, within the framework of the molecular biology thematic programme of the Semmelweis University, by the National Office for Innovation and Research (KH130355, and 2020-1.1.6-JOVO-2021-00013 "JOVO" to Z.P.). The study was performed in frame of the Premium Postdoctoral Fellowship Program of the Hungarian Academy of Sciences (PPD2018-016/2018 to D.C.). Z.P. and L.H. are supported by funds of the EU MSCA project CORVOS 860044 .

\section{Conflict of Interest}

T.M. is an Advisory Board member of AbbVie, BMS, Janssen-Cilag, Novartis, Pfizer, and Takeda. Other authors have no conflict of interest to declare.

\section{Acknowledgments}

We acknowledge the technical assistance of Márta Kókai, Éva Zsuzsanna Szendrei, Lászlóné Kertész, Edina Szabó, and Beáta Takács.

\section{References}

1 Guan WJ, Ni ZY, Hu Y, et al; China Medical Treatment Expert Group for Covid-19. Clinical characteristics of coronavirus disease 2019 in China. N Engl J Med 2020;382(18):1708-1720

2 Zhou F, Yu T, Du R, et al. Clinical course and risk factors for mortality of adult inpatients with COVID-19 in Wuhan, China: a retrospective cohort study. Lancet 2020;395(10229):1054-1062

3 Chen G, Wu D, Guo W, et al. Clinical and immunological features of severe and moderate coronavirus disease 2019. J Clin Invest 2020;130(05):2620-2629

4 Moore JB, June $\mathrm{CH}$. Cytokine release syndrome in severe COVID19. Science $2020 ; 368(6490): 473-474$

5 Helms J, Tacquard C, Severac F, et al; CRICS TRIGGERSEP Group (Clinical Research in Intensive Care and Sepsis Trial Group for Global Evaluation and Research in Sepsis) High risk of thrombosis in patients with severe SARS-CoV-2 infection: a multicenter prospective cohort study. Intensive Care Med 2020;46(06): 1089-1098

6 Levi M, Thachil J, Iba T, Levy JH. Coagulation abnormalities and thrombosis in patients with COVID-19. Lancet Haematol 2020;7 (06):e438-e440

7 Escher R, Breakey N, Lämmle B. Severe COVID-19 infection associated with endothelial activation. Thromb Res 2020;190:62

8 Goshua G, Pine AB, Meizlish ML, et al. Endotheliopathy in COVID19-associated coagulopathy: evidence from a single-centre, cross-sectional study. Lancet Haematol 2020;7(08):e575-e582

9 Rauch A, Labreuche J, Lassalle F, et al. Coagulation biomarkers are independent predictors of increased oxygen requirements in COVID-19. J Thromb Haemost 2020;18(11):2942-2953

10 Escher R, Breakey N, Lämmle B. ADAMTS13 activity, von Willebrand factor, factor VIII and D-dimers in COVID-19 inpatients. Thromb Res 2020;192:174-175

11 Bazzan M, Montaruli B, Sciascia S, Cosseddu D, Norbiato C, Roccatello D. Low ADAMTS 13 plasma levels are predictors of mortality in COVID-19 patients. Intern Emerg Med 2020;15(05): 861-863

12 Huisman A, Beun R, Sikma M, Westerink J, Kusadasi N. Involvement of ADAMTS13 and von Willebrand factor in thromboembolic events in patients infected with SARS-CoV-2. Int J Lab Hematol 2020;42(05):e211-e212
13 Martinelli N, Montagnana M, Pizzolo F, et al. A relative ADAMTS13 deficiency supports the presence of a secondary microangiopathy in COVID 19. Thromb Res 2020;193:170-172

14 Tiscia GL, Favuzzi G, De Laurenzo A, et al; CSS COVID-19 Group. Reduction of ADAMTS13 levels predicts mortality in SARS-CoV-2 patients. TH Open 2020;4(03):e203-e206

15 Delrue M, Siguret V, Neuwirth $M$, et al. von Willebrand factor/ADAMTS13 axis and venous thromboembolism in moderate-to-severe COVID-19 patients. Br J Haematol 2021;192(06): 1097-1100

16 Mancini I, Baronciani L, Artoni A, et al. The ADAMTS13-von Willebrand factor axis in COVID-19 patients. J Thromb Haemost 2021;19(02):513-521

17 Favaloro EJ, Henry BM, Lippi G. Increased VWF and decreased ADAMTS-13 in COVID-19: creating a milieu for (micro)thrombosis. Semin Thromb Hemost 2021;47(04):400-418

18 Philippe A, Chocron R, Gendron N, et al. Circulating Von Willebrand factor and high molecular weight multimers as markers of endothelial injury predict COVID-19 in-hospital mortality. Angiogenesis 2021;24(03):505-517

19 Rodríguez Rodríguez M, Castro Quismondo N, Zafra Torres D, Gil Alos D, Ayala R, Martinez-Lopez J. Increased von Willebrand factor antigen and low ADAMTS13 activity are related to poor prognosis in covid-19 patients. Int J Lab Hematol 2021;43(04):0152-0155

20 Sweeney JM, Barouqa M, Krause GJ, et al. Evidence for secondary thrombotic microangiopathy in COVID-19. medRxiv 2020. Doi: 10.1101/2020.10.20.20215608

21 von Meijenfeldt FA, Havervall S, Adelmeijer J, et al. Prothrombotic changes in patients with COVID-19 are associated with disease severity and mortality. Res Pract Thromb Haemost 2020;5(01): 132-141

22 Cugno M, Meroni PL, Gualtierotti R, et al. Complement activation and endothelial perturbation parallel COVID-19 severity and activity. J Autoimmun 2021;116:102560

23 Ward SE, Curley GF, Lavin M, et al; Irish COVID-19 Vasculopathy Study (ICVS) Investigators. Von Willebrand factor propeptide in severe coronavirus disease 2019 (COVID-19): evidence of acute and sustained endothelial cell activation. Br J Haematol 2021;192 (04):714-719

24 Varga Z, Flammer AJ, Steiger P, et al. Endothelial cell infection and endotheliitis in COVID-19. Lancet 2020;395(10234):1417-1418

25 Neubauer K, Zieger B. Endothelial cells and coagulation. Cell Tissue Res 2021. Doi: 10.1007/s00441-021-03471-2

26 Ekdahl KN, Teramura Y, Hamad OA, et al. Dangerous liaisons: complement, coagulation, and kallikrein/kinin cross-talk act as a linchpin in the events leading to thromboinflammation. Immunol Rev 2016;274(01):245-269

27 de Nooijer AH, Grondman I, Janssen NAF, et al; RCI-COVID-19 study group. Complement activation in the disease course of coronavirus disease 2019 and its effects on clinical outcomes. J Infect Dis 2021;223(02):214-224

28 Holter JC, Pischke SE, de Boer E, et al. Systemic complement activation is associated with respiratory failure in COVID-19 hospitalized patients. Proc Natl Acad Sci U S A 2020;117(40): 25018-25025

29 Sinkovits G, Mező B, Réti M, et al. Complement overactivation and consumption predicts in-hospital mortality in SARS-CoV-2 infection. Front Immunol 2021;12:663187

30 Ward PA. The harmful role of c5a on innate immunity in sepsis. J Innate Immun 2010;2(05):439-445

31 Gombos T, Makó V, Cervenak L, et al. Levels of von Willebrand factor antigen and von Willebrand factor cleaving protease (ADAMTS13) activity predict clinical events in chronic heart failure. Thromb Haemost 2009;102(03):573-580

32 Czúcz J, Schaffer G, Csuka D, et al. Endothelial cell function in patients with hereditary angioedema: elevated soluble E-selectin level during inter-attack periods. J Clin Immunol 2012;32(01): 61-69 
33 Maharaj S, Xue R, Rojan A. Thrombotic thrombocytopenic purpura (TTP) response following COVID-19 infection: Implications for the ADAMTS-13-von Willebrand factor axis. J Thromb Haemost 2021;19(04):1130-1132

34 Blasi A, von Meijenfeldt FA, Adelmeijer J, et al. In vitro hypercoagulability and ongoing in vivo activation of coagulation and fibrinolysis in COVID-19 patients on anticoagulation. J Thromb Haemost 2020;18(10):2646-2653

35 Henry BM, Benoit SW, de Oliveira MHS, Lippi G, Favaloro EJ, Benoit JL. ADAMTS13 activity to von Willebrand factor antigen ratio predicts acute kidney injury in patients with COVID-19: evidence of SARS-CoV-2 induced secondary thrombotic microangiopathy. Int J Lab Hematol 2021;43(Suppl 1):129-136

36 De Cristofaro R, Liuzzo G, Sacco M, Lancellotti S, Pedicino D, Andreotti F. Marked von Willebrand factor and factor VIII elevations in severe acute respiratory syndrome coronavirus-2-positive, but not severe acute respiratory syndrome coronavirus-2negative, pneumonia: a case-control study. Blood Coagul Fibrinolysis 2021;32(04):285-289

37 Joly BS, Darmon M, Dekimpe C, et al. Imbalance of von Willebrand factor and ADAMTS13 axis is rather a biomarker of strong inflammation and endothelial damage than a cause of thrombotic process in critically ill COVID-19 patients. J Thromb Haemost 2021;19(09):2193-2198

38 Doevelaar AAN, Bachmann M, Hölzer B, et al. von Willebrand factor multimer formation contributes to immunothrombosis in coronavirus disease 2019. Crit Care Med 2021;49(05):e512-e520

39 Pascreau T, Zia-Chahabi S, Zuber B, Tcherakian C, Farfour E, Vasse M. ADAMTS 13 deficiency is associated with abnormal distribution of von Willebrand factor multimers in patients with COVID19. Thromb Res 2021;204:138-140

40 Pinsky DJ, Naka Y, Liao $\mathrm{H}$, et al. Hypoxia-induced exocytosis of endothelial cell Weibel-Palade bodies. A mechanism for rapid neutrophil recruitment after cardiac preservation. J Clin Invest 1996;97(02):493-500

41 Bashir DA, Da Q, Pradhan S, et al. Secretion of von Willebrand factor and suppression of ADAMTS-13 activity by markedly high concentration of ferritin. Clin Appl Thromb Hemost 2021; 27:1076029621992128

42 Tomar B, Anders HJ, Desai J, Mulay SR. Neutrophils and neutrophil extracellular traps drive necroinflammation in COVID-19. Cells 2020;9(06):E1383

43 Zuo Y, Yalavarthi S, Shi H, et al. Neutrophil extracellular traps in COVID-19. JCI Insight 2020;5(11):138999

44 Yang J, Wu Z, Long $\mathrm{Q}$ et al. Insights into immunothrombosis: the interplay among neutrophil extracellular trap, von Willebrand factor, and ADAMTS13. Front Immunol 2020;11:610696

45 Sorvillo N, Mizurini DM, Coxon C, et al. Plasma peptidylarginine deiminase IV promotes VWF-platelet string formation and accelerates thrombosis after vessel injury. Circ Res 2019;125(05): 507-519
46 Wang Y, Chen J, Ling M, López JA, Chung DW, Fu X. Hypochlorous acid generated by neutrophils inactivates ADAMTS13: an oxidative mechanism for regulating ADAMTS13 proteolytic activity during inflammation. J Biol Chem 2015;290(03):1422-1431

47 Pillai VG, Bao J, Zander CB, et al. Human neutrophil peptides inhibit cleavage of von Willebrand factor by ADAMTS13: a potential link of inflammation to TTP. Blood 2016;128(01):110-119

48 Ward SE, Fogarty H, Karampini E, et al; Irish COVID-19 Vasculopathy Study (iCVS) investigators. ADAMTS13 regulation of VWF multimer distribution in severe COVID-19. J Thromb Haemost 2021;19(08):1914-1921

49 Dong JF, Moake JL, Nolasco L, et al. ADAMTS-13 rapidly cleaves newly secreted ultralarge von Willebrand factor multimers on the endothelial surface under flowing conditions. Blood 2002;100 (12):4033-4039

50 Bettoni S, Galbusera M, Gastoldi S, et al. Interaction between multimeric von Willebrand factor and complement: a fresh look to the pathophysiology of microvascular thrombosis. J Immunol 2017;199(03):1021-1040

51 Magro C, Mulvey JJ, Berlin D, et al. Complement associated microvascular injury and thrombosis in the pathogenesis of severe COVID-19 infection: a report of five cases. Transl Res 2020;220:1-13

52 Cugno M, Meroni PL, Gualtierotti R, et al. Complement activation in patients with COVID-19: a novel therapeutic target. J Allergy Clin Immunol 2020;146(01):215-217

53 Morigi M, Galbusera M, Gastoldi S, et al. Alternative pathway activation of complement by Shiga toxin promotes exuberant C3a formation that triggers microvascular thrombosis. J Immunol 2011;187(01):172-180

54 Ikeda K, Nagasawa K, Horiuchi T, Tsuru T, Nishizaka H, Niho Y. C5a induces tissue factor activity on endothelial cells. Thromb Haemost 1997;77(02):394-398

55 Tedesco F, Pausa M, Nardon E, Introna M, Mantovani A, Dobrina A. The cytolytically inactive terminal complement complex activates endothelial cells to express adhesion molecules and tissue factor procoagulant activity. J Exp Med 1997;185(09):1619-1627

56 Conway EM. Thrombomodulin and its role in inflammation. Semin Immunopathol 2012;34(01):107-125

57 Klos A, Tenner AJ, Johswich KO, Ager RR, Reis ES, Köhl J. The role of the anaphylatoxins in health and disease. Mol Immunol 2009;46 (14):2753-2766

58 Kawecki C, Lenting PJ, Denis CV. von Willebrand factor and inflammation. J Thromb Haemost 2017;15(07):1285-1294

59 Skendros P, Mitsios A, Chrysanthopoulou A, et al. Complement and tissue factor-enriched neutrophil extracellular traps are key drivers in COVID-19 immunothrombosis. J Clin Invest 2020;130 (11):6151-6157

60 Bonaventura A, Vecchié A, Dagna L, et al. Endothelial dysfunction and immunothrombosis as key pathogenic mechanisms in COVID-19. Nat Rev Immunol 2021;21(05):319-329 